UCB-PTH-02/20

LBNL-50199

\title{
A Complete Theory of Grand Unification in Five Dimensions
}

\author{
Lawrence J. Hall and Yasunori Nomura \\ Department of Physics, University of California, Berkeley, CA 94720, USA \\ Theoretical Physics Group, Lawrence Berkeley National Laboratory, Berkeley, CA 94720, USA
}

\begin{abstract}
A fully realistic unified theory is constructed, with $S U(5)$ gauge symmetry and supersymmetry both broken by boundary conditions in a fifth dimension. Despite the resulting explicit breaking of $S U(5)$ locally at a boundary of the dimension, when the size of the extra dimension is taken to be large precise predictions emerge for gauge coupling unification, $\alpha_{s}\left(M_{Z}\right)=0.118 \pm 0.003$, and for Yukawa coupling unification, $m_{b}\left(M_{Z}\right)=3.3 \pm 0.2 \mathrm{GeV}$. The $5 \mathrm{D}$ theory is then valid over a large energy interval from the compactification scale, $M_{c} \simeq 1 \times 10^{15} \mathrm{GeV}$, to the scale of strong coupling, $M_{s} \simeq 1 \times 10^{17} \mathrm{GeV}$. A complete understanding of the Higgs sector of the minimal supersymmetric standard model is given; with explanations for why the Higgs triplets are heavy, why the Higgs doublets are protected from a large tree-level mass, and why the $\mu$ and $B$ parameters are naturally generated to be of order the supersymmetry breaking scale. All sources of proton decay from operators of dimension four and five are forbidden, while a new origin for baryon number violating dimension six operators is found to be important. The exchange of the superheavy gauge boson, with a brane-localized kinetic energy interaction, leads to $\tau_{p} \approx 10^{34}$ years, with several branching ratios determined in terms of a single mixing parameter. The theory is only realistic for an essentially unique choice of matter location in the fifth dimension: the ten-plets of the first two generations must lie in the bulk, with all other matter located on the $S U(5)$ preserving boundary. Several aspects of flavor follow from this geometry: only the third generation possesses an $S U(5)$ mass relation, and the lighter two generations have only small mixings with the heaviest generation except for neutrinos. The entire superpartner spectrum is predicted in terms of only two free parameters. The squark and slepton masses have sizes determined by their location in the fifth dimension, allowing a significant experimental test of the detailed structure of the extra dimension. Lepton flavor violation is found to be generically large in higher dimensional unified theories with non-trivial matter geometries, providing soft supersymmetry breaking operators are local up to the compactification scale. In our theory this forces a common location for all three neutrinos, predicting large neutrino mixing angles. Rates for $\mu \rightarrow e \gamma, \mu \rightarrow e e e, \mu \rightarrow e$ conversion and $\tau \rightarrow \mu \gamma$ are larger in our theory than in conventional 4D supersymmetric grand unified theories, and, once superpartner masses are measured, these rates are completely determined in terms of two leptonic mixing angles. Proposed experiments probing $\mu \rightarrow e$ transitions will probe the entire interesting parameter space of our theory.
\end{abstract}




\section{Introduction}

Weak scale supersymmetry not only provides a framework for electroweak symmetry breaking, but also leads to a highly successful prediction for the unification of gauge couplings. If this picture of a supersymmetric desert is correct, the scale of gauge coupling unification heralds the threshold for some new unified physics; for example, conventional supersymmetric grand unified theories or string theory. We have recently introduced a new alternative for the unified physics, which we call Kaluza-Klein (KK) grand unification [1, 2]. In this framework the grand unified symmetry is realized in higher dimensions, but is explicitly broken locally by defect branes, and consequently does not appear as a symmetry of the low energy effective 4D theory. In general the gauge symmetry breaking associated with the local defects destroys gauge coupling unification; however, if the volume of the bulk is large, this symmetry breaking is diluted, restoring gauge coupling unification [1]. In all known 4D grand unified theories, the accuracy of the prediction for the QCD coupling from gauge coupling unification is limited because the unified threshold corrections cannot be computed. In KK grand unification, if the volume of the bulk is increased so that the theory becomes strongly coupled at the cutoff scale, the leading unified scale corrections can be computed, leading to a new level of precision for gauge coupling unification [2].

Several features of KK grand unified theories make them extremely attractive candidates for physics in the region of the unification scale. They incorporate the advances of conventional grand unified theories, such as charge quantization and the quantum numbers of the quarks and leptons, while overcoming their problematic features. In particular the orbifold boundary conditions automatically require that multiplets in the bulk are split in mass. This is particularly important for the gauge and Higgs multiplets, and provides an elegant origin for gauge symmetry breaking and for a large mass splitting between the Higgs triplets and doublets [3]. While the simplest 4D supersymmetric $S U(5)$ theory 41 is excluded, by too large a proton decay rate mediated by colored Higgsino exchange [5], in KK grand unified theories the form for the Higgsino mass matrix is determined by the KK mode expansion and automatically forbids proton decay from Higgsino exchange [1]. Finally, KK grand unification does not lead to fermion mass relations for all matter — no relations are expected for bulk matter [1, 6]. Hence there is a successful correlation: only the heavier fermions are expected to exhibit unified mass relations [2, 7, 8].

In Ref. [2] we have constructed a minimal theory of KK grand unification, which has $S U(5)$ gauge symmetry in 5D and provides a uniquely successful, high-precision prediction for the QCD coupling, $\alpha_{s}\left(M_{Z}\right)=0.118 \pm 0.004 \pm 0.003$, where the first uncertainty arises from the supersymmetric threshold and the second from the scale of strong coupling. It is only in this 
case that the unified scale corrections, from the KK towers of gauge and minimal Higgs sectors, give agreement with experiment $\alpha_{s}^{\exp }\left(M_{Z}\right)=0.117 \pm 0.002$ [9]. In this theory $R$ parity arises as a subgroup of a continuous $U(1)_{R}$ symmetry that is related to the $S U(2)_{R}$ symmetry of the bulk supersymmetry. This $U(1)_{R}$ symmetry forbids a mass operator for the Higgs fields, completing the solution to the doublet-triplet splitting problem, and forbids all proton decay from operators of dimension four and five. Furthermore, this theory is sufficiently tight that certain aspects of flavor must be related to the geometrical location of matter in the extra dimension. In particular the top quark resides on a brane while the up quark is in the bulk.

In this paper we pursue this 5D $S U(5)$ theory further, addressing two questions:

- Can the theory be made completely realistic?

- Can the theory be experimentally tested?

These two questions are closely related. Supersymmetry breaking is the major remaining additional ingredient needed for the theory to be fully realistic, and it is via the precise form of the soft supersymmetry breaking interactions that further tests of the theory are possible. Clearly there may be several ways to successfully incorporate supersymmetry breaking, and hence several versions of the fully realistic theory to test. In this paper we break supersymmetry by boundary conditions, using the same extra dimension that breaks the gauge symmetry [10]. This is highly economical, involving the vacuum expectation value of a field in the 5D gravity multiplet [11, and is highly predictive, since the most general such boundary condition involves just a single free parameter $\alpha$. Such supersymmetry breaking further constrains the location of matter because squarks and sleptons in the bulk acquire a tree-level mass $\tilde{m}=\alpha / R$, while those on the brane are massless at tree level. Combining previous constraints on matter location with considerations of superpartner induced flavor changing interactions, the location of every quark and lepton is determined, up to a two-fold ambiguity. In particular, the three $S U(5)$ five-plets, $F_{i}$, must either all be on the $S U(5)$ preserving brane, as shown in Fig. 2, or they must all be in the bulk. We will concentrate on the first case, since only then does a unified prediction for $m_{b} / m_{\tau}$ result. The second case is not uninteresting, since it leads to a geometric suppression of $m_{b} / m_{t}$, although we will find that some of this ratio must originate in $\tan \beta$.

Although we provide numerical predictions for this particular origin of supersymmetry breaking, many of the physical effects and signals we consider in this paper are much more general. These include contributions to $m_{b} / m_{\tau}$ from KK towers, an essential uniqueness of the matter location and its consequence of large mixing angles for neutrinos, superpartner masses reflecting the geometry of their locations, large lepton flavor violating signals, and a new origin and predictions for gauge boson mediated proton decay. In particular, we propose lepton flavor violation as a powerful and generic signal for KK grand unified theories with high mediation 
scales of supersymmetry breaking, and identify the general structure of soft supersymmetry breaking operators at the compactification scale by studying the flavor symmetry of the 5D gauge interactions.

The entire spectrum for the superpartners and the Higgs sector is predicted in our theory in terms of the supersymmetry breaking mass scale $\tilde{m}$ and the ratio of electroweak vacuum expectation values, $\tan \beta$. The predictions are characteristic of the underlying locations of each matter field in the extra dimension, and therefore provide a significant probe of the short distance structure of the theory. The combination of matter location and boundary condition supersymmetry breaking leads to flavor violation in the superpartner interactions. Large lepton flavor violating signals are predicted in terms of the parameters $\tilde{m}, \tan \beta$ and two flavor mixing angles $\theta_{12}^{e}$ and $\theta_{23}^{e}$. Future experiments probing $\mu \rightarrow e$ and $\tau \rightarrow \mu$ transitions could probe essentially all of the parameter space of the theory where electroweak symmetry breaking occurs naturally. Finally we give predictions from coupling constant unification for both $\alpha_{s}\left(M_{Z}\right)$ and $m_{b}\left(M_{Z}\right)$ which include corrections from KK towers at the unified scale and from superpartners at the weak scale.

Neutrino masses occur in our theory via the see-saw mechanism [12]. The masses of the right-handed neutrinos are governed by the breaking of a $U(1)_{X}$ gauge symmetry near the compactification scale. The neutrino flavor mixing angles are expected to be large because the neutrinos reside in the five-plets $F_{i}$, which all have a common location. Our theory contains a brane coupling between the two Higgs doublets and a gauge singlet field $X$ of the form $X H \bar{H}$. The supersymmetric dynamics which breaks $U(1)_{X}$ gauge symmetry determines $X$ to have vanishing vacuum expectation values. However, once supersymmetry breaking is included, a readjustment of the vacuum occurs so that $\langle X\rangle \approx \tilde{m}$ and $\left\langle F_{X}\right\rangle \approx \tilde{m}^{2}$, providing a natural origin for $\mu$ and $\mu B$ parameters 13 .

In sub-section 2.1 we review the basic features of our $S U(5)$ theory in 5D, paying particular attention to gauge coupling unification and $U(1)_{R}$ symmetry. In sub-section 2.2 we introduce boundary condition supersymmetry breaking, and give the form for the soft operators and the predictions for the soft supersymmetry breaking parameters at the weak scale. In section 3 we discuss several consequences of our theory: quark and lepton masses, supersymmetric threshold corrections to gauge coupling unification, Yukawa coupling unification, proton decay, and $R$ axions and axinos. While we work in the specific context of boundary condition supersymmetry breaking, some of our analyses, for example for proton decay and unified scale correction to $b / \tau$ unification, are completely independent of how supersymmetry is broken. In section $₫$ we study the general structure of flavor symmetries in KK grand unified theories and argue that large lepton flavor violation is a generic signature of these theories, providing that soft supersymmetry breaking operators are generated at or above the compactification scale. We then 
study the supersymmetric flavor violation induced in our theory, paying particular attention to the predictions in the lepton sector. In section 5 we discuss neutrino masses and the generation of the $\mu$ term, which are linked by the breaking of $U(1)_{X}$ gauge symmetry. Finally, in section 6 we discuss the variant of our theory where the $F_{i}$ are located in the bulk. Conclusions are drawn in section 7 .

\section{The Theory}

In this section we introduce our theory. In sub-section 2.1, we overview the 5D $S U(5) \mathrm{KK}$ grand unified theory of Ref. [2], discussing the symmetry structure and field content. We show that gauge coupling unification occurs, despite a point symmetry defect, and is in precise agreement with data. We explain solutions to the three outstanding problems of 4D supersymmetric grand unification: doublet-triplet splitting, proton decay, and fermion mass relations. We find that an $R$ symmetry, originating from the 5D supersymmetry, is crucial for a successful phenomenology.

In sub-section 2.2, we introduce supersymmetry breaking by boundary conditions following Ref. [10], leading to the usual soft supersymmetry breaking operators with coefficients determined by a single free parameter. Predictions for the supersymmetric particle spectrum are given, together with a brief discussion of collider phenomenology.

\subsection{Minimal Kaluza-Klein grand unification}

\subsubsection{Boundary conditions and restricted unified gauge symmetry}

We consider a 5D $S U(5)$ supersymmetric gauge theory compactified on an $S^{1} / Z_{2}$ orbifold. The $5 \mathrm{D}$ gauge multiplet $\mathcal{V}=\left\{A_{M}, \lambda, \lambda^{\prime}, \sigma\right\}$ consists of a 5D vector field, $A_{M}$, two gauginos, $\lambda$ and $\lambda^{\prime}$, and a real scalar, $\sigma$. Compactification on $S^{1} / Z_{2}$ is obtained by identifying the fifth coordinate $y$ under the two operations, $\mathcal{Z}: y \rightarrow-y$ and $\mathcal{T}: y \rightarrow y+2 \pi R$. The resulting space is a line interval $y \in[0, \pi R]$, with boundaries at $y=0$ and $\pi R$. Boundary conditions are chosen so that the orbifold reflection $\mathcal{Z}$ reduces $5 \mathrm{D} N=1$ supersymmetry to $4 \mathrm{D} N=1$ supersymmetry and preserves $S U(5)$, while the translation $\mathcal{T}$ breaks $S U(5)$ by the action of $P=\operatorname{diag}(+,+,+,-,-)$ on a 5-plet. In particular, the boundary conditions of the gauge multiplet are given by

$$
\left(\begin{array}{c}
V^{(p)} \\
\Sigma^{(p)}
\end{array}\right)\left(x^{\mu}, y\right)=\left(\begin{array}{c}
V^{(p)} \\
-\Sigma^{(p)}
\end{array}\right)\left(x^{\mu},-y\right)=p\left(\begin{array}{c}
V^{(p)} \\
\Sigma^{(p)}
\end{array}\right)\left(x^{\mu}, y+2 \pi R\right)
$$

where we have used the $4 \mathrm{D} N=1$ superfield language, $\mathcal{V}=\{V, \Sigma\}: V\left(A_{\mu}, \lambda\right)$ and $\Sigma((\sigma+$ $\left.\left.i A_{5}\right) / \sqrt{2}, \lambda^{\prime}\right)$ are $4 \mathrm{D}$ vector and chiral superfields in the adjoint representation. The standard

1 This is equivalent to the boundary conditions of Ref. [3] described in terms of $\mathcal{Z}$ and $\mathcal{Z}^{\prime}=\mathcal{Z} \mathcal{T}$ as $S^{1} /\left(Z_{2} \times Z_{2}^{\prime}\right)$. 


\begin{tabular}{|c|c|c|c|c|}
\hline$(\mathcal{Z}, \mathcal{T})$ & gauge and Higgs fields & bulk matter fields & KK modes & 4D masses \\
\hline$(+,+)$ & $V_{321}, H_{D}, \bar{H}_{D}$ & $T_{U, E}, T_{Q}^{\prime}, F_{D}, F_{L}^{\prime}$ & $\cos [n y / R]$ & $n / R$ \\
$(+,-)$ & $V_{X}, H_{T}, \bar{H}_{T}$ & $T_{Q}, T_{U, E}^{\prime}, F_{L}, F_{D}^{\prime}$ & $\cos [(n+1 / 2) y / R]$ & $(n+1 / 2) / R$ \\
$(-,+)$ & $\Sigma_{321}, H_{D}^{c}, \bar{H}_{D}^{c}$ & $T_{U, E}^{c}, T_{Q}^{\prime c}, F_{D}^{c}, F_{L}^{\prime c}$ & $\sin [(n+1) y / R]$ & $(n+1) / R$ \\
$(-,-)$ & $\Sigma_{X}, H_{T}^{c}, \bar{H}_{T}^{c}$ & $T_{Q}^{c}, T_{U, E}^{\prime c}, F_{L}^{c}, F_{D}^{\prime c}$ & $\sin [(n+1 / 2) y / R]$ & $(n+1 / 2) / R$ \\
\hline
\end{tabular}

Table 1: The transformation properties for the bulk fields under the orbifold reflection and translation. Here, we have used the $4 \mathrm{D} N=1$ superfield language. The fields written in the $(\mathcal{Z}, \mathcal{T})$ column, $\varphi$, obey the boundary condition $\varphi(y)=\mathcal{Z} \varphi(-y)=\mathcal{T} \varphi(y+2 \pi R)$. The modes and masses for the corresponding $\mathrm{KK}$ towers are also given $(n=0,1, \cdots)$.

model gauge multiplets $\left(V_{321}, \Sigma_{321}\right) \equiv\left(V^{(+)}, \Sigma^{(+)}\right)$have positive eigenvalues for the $P$ matrix, $p=1$, while the broken $S U(5)$ gauge multiplets $\left(V_{X}, \Sigma_{X}\right) \equiv\left(V^{(-)}, \Sigma^{(-)}\right)$have negative eigenvalues, $p=-1$. After the KK decomposition, only the minimal supersymmetric standard model (MSSM) gauge multiplets, $V_{321}$, have massless modes, and all the other modes have masses of the order of the compactification scale $M_{c} \equiv 1 / R$, as summarized in Table 1. We will see later that $M_{c}$ must be very large, of order $10^{15} \mathrm{GeV}$, but it differs from the conventional unification mass scale $M_{u} \simeq 2 \times 10^{16} \mathrm{GeV}$.

What is the gauge symmetry of this theory? While the low-energy $4 \mathrm{D}$ theory has only the standard model gauge symmetry, the original 5D theory has a larger gauge symmetry. We find that this gauge symmetry is $S U(5)$ but with the gauge transformation parameters obeying the same boundary conditions as the corresponding 4D gauge fields:

$$
\xi^{(p)}\left(x^{\mu}, y\right)=\xi^{(p)}\left(x^{\mu},-y\right)=p \xi^{(p)}\left(x^{\mu}, y+2 \pi R\right)
$$

which we refer to as restricted gauge symmetry [四]. The KK expansions for the standard model gauge parameters, $\xi^{(+)}=\xi_{321}$, and $S U(5) /\left(S U(3)_{C} \times S U(2)_{L} \times U(1)_{Y}\right)$ ones, $\xi^{(-)}=\xi_{X}$, are

$$
\begin{aligned}
\xi_{321}\left(x^{\mu}, y\right) & =\sum_{n=0}^{\infty} \xi_{321}^{n}\left(x^{\mu}\right) \cos \frac{n y}{R} \\
\xi_{X}\left(x^{\mu}, y\right) & =\sum_{n=0}^{\infty} \xi_{X}^{n}\left(x^{\mu}\right) \cos \frac{(n+1 / 2) y}{R} .
\end{aligned}
$$

Since $\xi_{X}$ always vanish at $y=\pi R$, the gauge symmetry is reduced to $S U(3)_{C} \times S U(2)_{L} \times U(1)_{Y}$ on this point, while the full $S U(5)$ symmetry is operative in all the other points in the extra dimension, as depicted in Fig. 1.

This structure allows the introduction of three types of fields: 4D $N=1$ superfields localized on the $y=0$ brane in representations of $S U(5), 4 \mathrm{D} N=1$ superfields on the $y=\pi R$ brane 


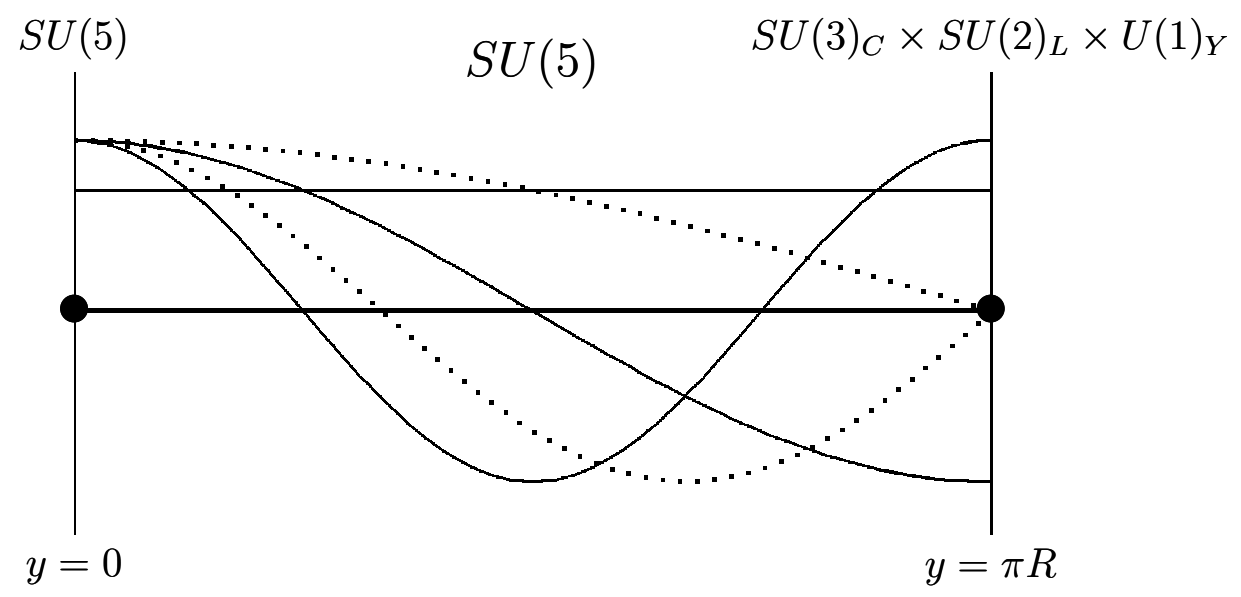

Figure 1: In the fifth dimension, space is a line segment bounded by branes at $y=0$ and at $y=\pi R$. Here, solid and dotted lines represent the profiles of gauge transformation parameters $\xi_{321}$ and $\xi_{X}$, respectively. Because $\xi_{X}(y=\pi R)=0$, explicit point defect symmetry breaking occurs at the $y=\pi R$ brane, which only respects $S U(3)_{C} \times S U(2)_{L} \times U(1)_{Y}$ gauge symmetry.

in representations of the standard model gauge group, and bulk fields forming 5D $N=1$ supermultiplets and representations of $S U(5)$. In particular, we can introduce a hypermultiplet $\mathcal{H}_{\Phi}=\left\{\phi, \phi^{c}, \psi, \psi^{c}\right\}$ in the bulk, which consists of two complex scalars, $\phi$ and $\phi^{c}$, and two Weyl fermions, $\psi$ and $\psi^{c}$. The boundary conditions for hypermultiplets are given by

$$
\left(\begin{array}{c}
\Phi^{(p)} \\
\Phi^{c(p)}
\end{array}\right)\left(x^{\mu}, y\right)=\left(\begin{array}{c}
\Phi^{(p)} \\
-\Phi^{c(p)}
\end{array}\right)\left(x^{\mu},-y\right)=p \eta_{\Phi}\left(\begin{array}{c}
\Phi^{(p)} \\
\Phi^{c(p)}
\end{array}\right)\left(x^{\mu}, y+2 \pi R\right),
$$

where we have used the $4 \mathrm{D} N=1$ superfield language, $\mathcal{H}_{\Phi}=\left\{\Phi, \Phi^{c}\right\}: \Phi(\phi, \psi)$ and $\Phi^{c}\left(\phi^{c}, \psi^{c}\right)$ are $4 \mathrm{D}$ chiral superfields. An overall factor $\eta_{\Phi}$ appears in the orbifold translation, which can be chosen to be $\eta_{\Phi}= \pm 1$ for each hypermultiplet.

The two Higgs doublets of the MSSM are introduced in the bulk as two hypermultiplets $\mathcal{H}_{H}=\left\{H, H^{c}\right\}$ and $\mathcal{H}_{\bar{H}}=\left\{\bar{H}, \bar{H}^{c}\right\}$, which transform as $\mathbf{5}$ and $\overline{\mathbf{5}}$ under $S U(5)$. The boundary conditions are given by Eq. (5) with $\eta_{H}=\eta_{\bar{H}}=-1$, so that we have massless Higgs doublets. (In the present notation, $H^{(+)}$and $H^{(-)}\left(\bar{H}^{(+)}\right.$and $\left.\bar{H}^{(-)}\right)$represent triplet and doublet components, $H_{T}$ and $H_{D}\left(\bar{H}_{T}\right.$ and $\left.\bar{H}_{D}\right)$, of $H(\bar{H})$, respectively.) The resulting KK towers are summarized in Table 1. These Higgs KK towers do not have zero modes for the color triplet states [3]. Moreover, since the mass term of the KK excitations takes the form $H H^{c}+\bar{H} \bar{H}^{c}$ rather than $H \bar{H}$, the exchange of triplet states does not lead to proton decay from dimension five operators [1]. At low energies, only the two Higgs doublets of the MSSM, $H_{D}$ and $\bar{H}_{D}$, remain from the Higgs KK towers. 
The quarks and leptons are introduced either in the bulk or on the $S U(5)$ brane at $y=0$, to preserve the $S U(5)$ understanding of matter quantum numbers [1]. If quarks and leptons are on the brane, they fill out 4D chiral multiplets which are $\mathbf{1 0}$ or $\overline{\mathbf{5}}$ representations of $S U(5): T$ and $F$. On the other hand, if quarks and leptons are in the bulk, they arise from hypermultiplets: $\left\{T, T^{c}\right\}+\left\{T^{\prime}, T^{\prime c}\right\}$ with $\eta_{T}=-\eta_{T^{\prime}}=1$ and $\left\{F, F^{c}\right\}+\left\{F^{\prime}, F^{\prime c}\right\}$ with $\eta_{F}=-\eta_{F^{\prime}}=1$. We then find from Eq. (5) that a generation $q, u, d, l, e$ arises from the zero modes of bulk fields $T(u, e), T^{\prime}(q), F(d)$ and $F^{\prime}(l)$. (The tower structure for these fields is given in Table 11. Note that $T^{(+)}=T_{U, E}, T^{(-)}=T_{Q}, F^{(+)}=F_{D}, F^{(-)}=F_{L}$, and similarly for $T^{\prime}$ and $F^{\prime}$, where $T_{Q, U, E}\left(F_{D, L}\right)$ are the components of $T(F)$ decomposed into irreducible representations of the standard model gauge group.) We can choose where we put quarks and leptons for each $S U(5)$ representation, 10 and $\overline{\mathbf{5}}$, in each generation. Thus, at this stage, we have $\left(2^{2}\right)^{3}=64$ different choices for the configuration of matter. We will identify the most attractive matter configuration later in this section.

\subsubsection{Gauge coupling unification from strong coupling}

So far, we have demonstrated how the orbifold compactification of the $5 \mathrm{D} S U(5)$ theory leads to the gauge group and matter content of the MSSM in the 4D effective theory below $M_{c}$. However, since $S U(5)$ is explicitly broken by boundary conditions, it is not obvious that this theory preserves successful gauge coupling unification. In fact, we find that gauge coupling unification is generically destroyed due to the presence of local $S U(5)$ breaking on the $y=\pi R$ brane. To see this, let us consider the effective field theory above $M_{c}$. Since the higher dimensional gauge theory is non-renormalizable, this effective theory must be cut off at some scale $M_{s}$, where the theory is embedded into a more fundamental theory such as string theory. At the scale $M_{s}$, the most general effective action for the gauge kinetic terms is

$$
S=\int d^{4} x d y\left[\frac{1}{g_{5}^{2}} F^{2}+\delta(y) \frac{1}{\tilde{g}^{2}} F^{2}+\delta(y-\pi R) \frac{1}{\tilde{g}_{a}^{2}} F_{a}^{2}\right]
$$

where the first and second terms are $S U(5)$-invariant bulk and brane gauge kinetic energies, while the third term represents non-unified kinetic operators located on the $y=\pi R$ brane ( $a=1,2,3$ represents the standard model gauge groups). This form is ensured by the restricted gauge symmetry, regardless of the unknown ultraviolet physics above $M_{s}$. The standard model gauge couplings in the equivalent KK theory, $g_{a}$, are obtained by integrating over the extra dimension:

$$
\frac{1}{g_{a}^{2}}=\frac{\pi R}{g_{5}^{2}}+\frac{1}{\tilde{g}_{a}^{2}},
$$

where the contribution from $\tilde{g}$ has been absorbed into a shift of the $\tilde{g}_{a}$. This shows that $g_{a}$ depend on the coefficients of the localized kinetic operators, $\tilde{g}_{a}$, and are not universal at the 
scale $M_{s}$. However, this difficulty is overcome by requiring the extra dimension to have a large volume [1]. Writing $g_{5}^{2}=\gamma / M_{s}$ and $\tilde{g}_{a}^{2}=\gamma_{a}$, we find that the non-universal term is suppressed compared with the universal term by a volume factor $\left(\gamma / \gamma_{a}\right)\left(1 / \pi M_{s} R\right)$. Therefore, by making the extra dimension large $\left(\pi R M_{s}\right.$ large), gauge coupling unification is recovered.

How large should we take the extra dimension? It depends on the unknown coefficients $\gamma$ and $\gamma_{a}$. In the extreme case of $\gamma_{a} \ll \gamma$, we even cannot recover gauge coupling unification by making the extra dimension large. In Ref. [2], we have introduced a framework which removes these concerns and makes KK grand unification more reliable and predictive. The crucial new ingredient is the assumption that the gauge interaction is strongly coupled at the cutoff scale $M_{s}$ [8]. While the theory is weakly coupled at $M_{c}$, it becomes strongly coupled at higher energies since a higher dimensional gauge coupling has negative mass dimensions. In the 4D picture this follows because the loop expansion parameter of the theory (the strength of the gauge interaction) is given by the usual loop factor, $C\left(g^{2} / 16 \pi^{2}\right)$, times the number of KK states available at the energy $E, N_{\mathrm{KK}}(E) \simeq\left(E / M_{c}\right)$, where $C$ represents a group theoretical factor $(C \simeq 5)$. We require this loop expansion parameter to be 1 at $M_{s}: C\left(g^{2} / 16 \pi^{2}\right)\left(M_{s} / M_{c}\right) \simeq 1$. Although $g$ itself also depends on the energy $E$, its evolution is slow up to energies very close to $M_{s}$ so that we may take $g$ to be the $4 \mathrm{D}$ gauge coupling at $M_{c}, g \simeq 0.7$, to estimate $M_{s} / M_{c}$, which gives $M_{s} / M_{c} \simeq 300 / C$. This strong coupling requirement has the following virtues. First, it allows us to estimate $\gamma$ and $\gamma_{a}$ by requiring that all the loop diagrams contribute equally at the scale $M_{s}$. By carefully evaluating expansion parameters, we find $\gamma \simeq 16 \pi^{3} / C$ and $\gamma_{a} \simeq 16 \pi^{2} / C_{a}$, excluding the unwanted situation $\gamma_{a} \ll \gamma$. This argument is quite similar to the case of the usual chiral theory of mesons: all the operator coefficients at the QCD scale $\Lambda$ are estimated to be products of appropriate powers of $4 \pi$ and $\Lambda$ by requiring that all the loop diagrams contribute equally [14]. Substituting these estimates into Eq. (7), we obtain

$$
\frac{1}{g_{a}^{2}} \simeq \frac{C M_{s} R}{16 \pi^{2}}+\frac{C_{a}}{16 \pi^{2}},
$$

at the scale $M_{s}$, where $C \simeq C_{a} \simeq 5$. To obtain $g_{a} \simeq 0.7$ requires $M_{s} R \simeq 60$, so that the non-unified contribution from unknown ultraviolet physics is suppressed to be a negligible level (less than a $1 \%$ correction to $g_{a}$ ). We adopt this strong coupling scenario in the rest of the paper.

\subsubsection{Consequences of the extra dimension being large}

The presence of a moderately large extra dimension, $M_{s} R=O(100)$, has several important consequences. First of all, the running of the gauge couplings between $M_{s}$ and $M_{c}$ gives a nonnegligible contribution to the prediction of the QCD coupling. In the energy interval between $M_{s}$ and $M_{c}$, the gauge couplings receive both power-law and logarithmic contributions. However, 
the leading power-law piece comes from the renormalization of the bulk gauge coupling and thus must be universal due to the restricted gauge symmetry. On the other hand, the logarithmic contributions come from the runnings of $4 \mathrm{D}$ gauge kinetic terms localized on the branes and can be different for $S U(3)_{C}, S U(2)_{L}$ and $U(1)_{Y}$. This means that the differences of the three gauge couplings evolve logarithmically above the compactification scale, although the gauge couplings themselves receive power law corrections [1, 15]. Since the beta-function coefficients for the relative runnings above $M_{c}$ are different from the MSSM beta-function coefficients, the prediction of minimal KK grand unification for the QCD coupling, $\alpha_{s}^{\mathrm{KK}}$, is different from the prediction of the single scale unification, $\alpha_{s}^{\mathrm{SGUT}, 0}$. The difference $\delta \alpha_{s} \equiv \alpha_{s}^{\mathrm{KK}}-\alpha_{s}^{\mathrm{SGUT}, 0}$ is given by

$$
\delta \alpha_{s} \simeq-\frac{3}{7 \pi} \alpha_{s}^{2} \ln \frac{M_{s}}{M_{c}^{\prime}}
$$

where $M_{c}^{\prime} \equiv M_{c} / \pi$ [2]. An important point is that $\delta \alpha_{s}$ is dominated by the calculable contribution coming from the energy interval between $M_{s}$ and $M_{c}^{\prime}$, since it gives a non-universal correction to $1 / g_{a}$ by an amount of order $\simeq\left(C_{a} / 16 \pi^{2}\right) \ln \left(M_{s} / M_{c}^{\prime}\right)$ which is larger than that from unknown ultraviolet physics, $\simeq\left(C_{a} / 16 \pi^{2}\right)$, by a factor of $\ln \left(M_{s} / M_{c}^{\prime}\right)$. The other uncertainties are also under control: the dependence of $\delta \alpha_{s}$ on $M_{s} / M_{c}^{\prime}$ is weak, and the effect from the strong coupling physics around $M_{s}$ is small [8]. Therefore, we obtain $\delta \alpha_{s} \approx-0.01$ from $M_{s} / M_{c}^{\prime} \approx 100$, eliminating the discrepancy between the usual supersymmetric prediction and data. The compactification scale $M_{c}^{\prime}$ is given by

$$
M_{c}^{\prime}=M_{u}\left(\frac{M_{c}^{\prime}}{M_{s}}\right)^{5 / 7} .
$$

Using $M_{u} \simeq 2 \times 10^{16} \mathrm{GeV}$ and $M_{s} / M_{c}^{\prime} \simeq 16 \pi^{3} / g^{2} C \simeq 200$, we obtain $M_{c}^{\prime} \simeq 5 \times 10^{14} \mathrm{GeV}$ and $M_{s} \simeq 1 \times 10^{17} \mathrm{GeV}$. These values become important when we discuss gauge and Yukawa coupling unifications in section 3 .

The second important consequence of the large dimension is that it explains part of the observed structure of fermion masses. Yukawa interactions are forbidden by 5D supersymmetry from appearing in the bulk Lagrangian, and hence must be brane localized. They are located on the $y=0$ brane

$$
S=\int d^{4} x d y \delta(y)\left[\int d^{2} \theta\left(y_{T} \hat{T} \hat{T} H+y_{F} \hat{T} \hat{F} \bar{H}\right)+\text { h.c. }\right]
$$

where $\hat{T}(\hat{F})$ runs over all the matter chiral superfields in the $\mathbf{1 0}(\overline{\mathbf{5}})$ representation: branelocalized $T(F)$ and bulk $T$ and $T^{\prime}\left(F\right.$ and $\left.F^{\prime}\right)$. Since the full $S U(5)$ symmetry is operative at $y=0$, these Yukawa couplings must respect the $S U(5)$ symmetry. This means that, if quarks and leptons are located on the brane, they respect $S U(5)$ mass relations. The resulting $4 \mathrm{D}$ 
Yukawa couplings are suppressed by a factor of $1 /\left(M_{s} R\right)^{1 / 2} \approx 0.1$ as the Higgs wavefunctions are spread out over the bulk. On the other hand, if quarks and leptons are in the bulk, $u, e$ and $q(d$ and $l)$ arise from different hypermultiplets $\mathcal{H}_{T}$ and $\mathcal{H}_{T^{\prime}}\left(\mathcal{H}_{F}\right.$ and $\left.\mathcal{H}_{F^{\prime}}\right)$. Therefore, they do not respect $S U(5)$ mass relations because the down-type quark and charged lepton masses come from different couplings, which are not related by the $S U(5)$ symmetry.2 Moreover, since the matter wavefunctions are also spread out in the extra dimension, the resulting 4D Yukawa couplings receive a stronger suppression, by a factor of $1 /\left(M_{s} R\right)^{3 / 2} \approx 10^{-3}$, than in the case of brane matter. Thus we find a clearly successful correlation between the mass of the fermion and whether it has $S U(5)$ mass relations - heavier fermions display $S U(5)$ mass relations while lighter ones do not. Obviously, Yukawa couplings involving both bulk and brane matter receive a suppression factor of $1 /\left(M_{s} R\right)$.

The location of some matter is determined because the extra dimension is "large". Since our theory has $M_{c} \approx 10^{15} \mathrm{GeV}$, the $X$ gauge bosons are considerably lighter, of mass about $10^{15} \mathrm{GeV}$, than in the case of $4 \mathrm{D}$ supersymmetric grand unification. This makes dimension six proton decay a non-trivial issue in our theory; for instance, if all the matter fields were localized on the brane, the $X$ gauge boson exchange would induce proton decay at too rapid a rate. We find that this rapid proton decay is avoided if the quarks and leptons of the first generation coming from a $\mathbf{1 0}$ representation are bulk fields, since then $q$ and $u, e$ come from different hypermultiplets and the broken gauge boson exchange does not lead to proton decay. We will say that $T_{1}$ is in the bulk, although we really mean the combination $\left\{T_{1}, T_{1}^{c}\right\}+\left\{T_{1}^{\prime}, T_{1}^{\prime c}\right\}$. On the other hand, the top quark must arise from a brane field $T_{3}$. If the top quark were a bulk mode, it would have a mass suppressed by a factor of $1 /\left(M_{s} R\right)^{3 / 2}$ giving too light a top quark, even in the case that the Yukawa interaction is strong at $M_{s}$. With $T_{3}$ on the brane, strong coupling leads to a top Yukawa coupling of the low energy theory of $4 \pi /\left(M_{s} R\right)^{1 / 2} \approx 1$, giving a top quark mass of the observed size. Thus, given the existence of the large dimension of size $M_{s} R=O(100)$, we are able to derive the location of both the first and third generation 10's, and we find that at least some aspects of flavor physics are associated with the geometry of the extra dimension, and with strong coupling. Further consequences of the large size of the fifth dimension, for example for gaugino mass relations and Yukawa coupling unification, are discussed in later sections.

We now proceed further with matter geography by considering fermion mass relations. The location of $F_{3}$ determines whether we have $b / \tau$ Yukawa unification, which gives a successful prediction for $m_{b} / m_{\tau}$ at $O(10 \%)$ level in supersymmetric grand unified theories [16]. For most of this paper we choose to put $F_{3}$ on the $y=0$ brane to preserve $b / \tau$ unification. (The theory

\footnotetext{
${ }^{2}$ We could also introduce Yukawa couplings for bulk matter on the $y=\pi R$ brane, which do not respect the $S U(5)$ symmetry.
} 
without $b / \tau$ unification is discussed in section 6.) On the other hand, since the $S U(5)$ mass relation for $s / \mu$ does not work, either $T_{2}$ or $F_{2}$ must be located in the bulk. Summarizing, we have derived the locations of $T_{1}, T_{3}$, and $F_{3}$ by considering dimension six proton decay, the size of the top Yukawa coupling, and $b / \tau$ Yukawa unification. We have also found that either $T_{2}$ or $F_{2}$ must be in the bulk to avoid an unwanted $S U(5)$ mass relation for $s / \mu$. Therefore, we are left with $2^{3}-1=7$ possibilities for the matter location at this stage, corresponding to choices for the locations of $T_{2}, F_{2}$, and $F_{1}$. Further determination must await the introduction of supersymmetry breaking in the next sub-section.

\subsection{4 $U(1)_{R}$ symmetry}

We here discuss the important issue of what further brane-localized operators can be introduced in the theory. The 5D restricted gauge symmetry alone allows many unwanted operators on the branes. For instance, the operators $[H \bar{H}]_{\theta^{2}}$ and $[F H]_{\theta^{2}}$ give a large mass, of order the unified scale, for the Higgs doublets destroying the solution to the doublet-triplet splitting problem, $[T F F]_{\theta^{2}}$ causes disastrous dimension four proton decay, and $[T T T F]_{\theta^{2}}$ induces too rapid dimension five proton decay. In addition, if matter is located in the bulk, $S U(5)$ non-invariant operators on the $y=\pi R$ brane, such as $\left[T_{Q} T_{Q} \bar{H}_{T}^{c}\right]_{\theta^{2}}$ and $\left[T_{Q} F_{L} H_{T}^{c}\right]_{\theta^{2}}$, reintroduce the problem of dimension five proton decay mediated by colored Higgsino exchange. Remarkably, however, the structure of the theory allows a mechanism that simultaneously suppresses all of these unwanted operators [1]. Since the bulk Lagrangian has higher dimensional supersymmetry, it possesses an $S U(2)_{R}$ symmetry. It also has an $S U(2)_{H}$ flavor symmetry rotating the two Higgs hypermultiplets in the bulk. After orbifolding, these two $S U(2)$ symmetries are broken to two $U(1)$ symmetries, one from $S U(2)_{R}$ and one from $S U(2)_{H}$. A particularly interesting symmetry is the diagonal subgroup of these $U(1)$ symmetries, which we call $U(1)_{R}$ symmetry since it is an $R$ symmetry rotating the Grassmann coordinate of the low energy $4 \mathrm{D} N=1$ supersymmetry. We can extend this bulk $U(1)_{R}$ symmetry to the full theory by assigning appropriate charges to the brane-localized quark and lepton superfields, and use it to constrain possible forms of brane-localized operators. The resulting $U(1)_{R}$ charges are given in Table 2, where $T$ and $F$ represent both brane and bulk matter (primed fields have the same charges as unprimed fields). Imposing this $U(1)_{R}$ symmetry on the theory, we can forbid unwanted operators while keeping the Yukawa couplings. Proton decay from operators of dimension four and five are prohibited, and all $R$-parity violating operators are absent since $U(1)_{R}$ contains the usual $R$ parity as a discrete subgroup. The $U(1)_{R}$ symmetry also forbids a bulk mass term for the Higgs hypermultiplets, $\left[H \bar{H}-H^{c} \bar{H}^{c}\right]_{\theta^{2}}$, which would remove the Higgs doublets from the low energy theory and reintroduce dimension five proton decay from colored Higgsino exchange. Therefore, the $U(1)_{R}$ symmetry provides a complete solution to the doublet-triplet and proton decay problems. 


\begin{tabular}{|c|cc|cccc|cccc|}
\hline & $V$ & $\Sigma$ & $H$ & $H^{c}$ & $H$ & $H^{c}$ & $T$ & $T^{c}$ & $F$ & $F^{c}$ \\
\hline$U(1)_{R}$ & 0 & 0 & 0 & 2 & 0 & 2 & 1 & 1 & 1 & 1 \\
\hline
\end{tabular}

Table 2: $U(1)_{R}$ charges for $4 \mathrm{D}$ vector and chiral superfields.

The above $U(1)_{R}$ is broken to its $R$-parity subgroup by supersymmetry breaking, introduced in the next sub-section. Since the breaking scale is small, however, it will not reintroduce the problem of proton decay. The presence of an $R$ symmetry broken only through supersymmetry breaking effects is also important for generating the supersymmetric mass term for the two Higgs doublets (the $\mu$ term) at the correct size of the order of the weak scale [13].

\subsection{Supersymmetry breaking from boundary conditions}

Having obtained a unified theory free from the problems of usual supersymmetric grand unification, we now introduce supersymmetry breaking into the theory. There are two natural ways of introducing supersymmetry breaking in theories with unified scale extra dimensions: one is through a supersymmetry breaking expectation value of some brane-localized 4D field [17, 18] and the other is through boundary conditions [10]. In the former case, there is a $4 \mathrm{D}$ chiral superfield $Z$ whose highest component, $F_{Z}$, has a non-vanishing vacuum expectation value. However, in our theory some of the lightest two generations of matter propagate in the bulk, so that this way of introducing supersymmetry breaking leads to the supersymmetric flavor problem through direct couplings such as $\left[Z^{\dagger} Z T_{i}^{\dagger} T_{j}\right]_{\theta^{2} \bar{\theta}^{2}}$. Therefore, we choose the latter case where supersymmetry is broken by boundary conditions.

One important feature of boundary condition breaking is that all of the supersymmetry breaking parameters are completely specified in terms of a single continuous parameter $\alpha$ [19]. The supersymmetry breaking is introduced by imposing the boundary conditions such that, under the orbifold translation $\mathcal{T}: y \rightarrow y+2 \pi R$, the component fields are rotated by a $U(1)$ subgroup of $S U(2)_{R}$ which does not commute with the orbifold reflection $\mathcal{Z}$; the angle of the rotation is parameterized by $\alpha$. Since $A_{M}$ and $\sigma$ are singlet under $S U(2)_{R}$, only the two gauginos, $\lambda$ and $\lambda^{\prime}$, are subject to the above rotation in the gauge multiplet. Similarly, for hypermultiplets, only scalar components, $\phi$ and $\phi^{c}$, receive the rotation. Then, without a loss of generality, we can take the boundary conditions for the gauginos and scalar components of hypermultiplets as

$$
\left(\begin{array}{c}
\lambda^{(p)} \\
\lambda^{\prime(p)}
\end{array}\right)\left(x^{\mu}, y\right)=\left(\begin{array}{c}
\lambda^{(p)} \\
-\lambda^{\prime(p)}
\end{array}\right)\left(x^{\mu},-y\right)=e^{2 \pi i \alpha \sigma_{2}} p\left(\begin{array}{c}
\lambda^{(p)} \\
\lambda^{\prime(p)}
\end{array}\right)\left(x^{\mu}, y+2 \pi R\right)
$$


and

$$
\left(\begin{array}{c}
\phi^{(p)} \\
\phi^{c(p) \dagger}
\end{array}\right)\left(x^{\mu}, y\right)=\left(\begin{array}{c}
\phi^{(p)} \\
-\phi^{c(p) \dagger}
\end{array}\right)\left(x^{\mu},-y\right)=e^{2 \pi i \alpha \sigma_{2}} p \eta_{\Phi}\left(\begin{array}{c}
\phi^{(p)} \\
\phi^{c(p) \dagger}
\end{array}\right)\left(x^{\mu}, y+2 \pi R\right)
$$

respectively, where $\sigma_{1,2,3}$ are the Pauli spin matrices. All the other component fields obey the same boundary conditions as before: Eqs. (11, 5).

After KK decomposition, the above boundary conditions generate soft supersymmetry breaking masses of order $\alpha / R$. Since $1 / R \approx 10^{15} \mathrm{GeV}, \alpha$ must be an extremely small number, $\alpha \approx 10^{-13}$. However, this does not mean that we need a fine tuning to obtain supersymmetry breaking masses of the order of the weak scale. In fact, it has been shown that the above supersymmetry breaking twist by $\alpha$ is equivalent to having a supersymmetry breaking vacuum expectation value for an auxiliary field in the 5D gravity multiplet [11]. In other words, by making a suitable gauge transformation that depends on the coordinate $y$, we can always go to the basis where the boundary conditions do not have any supersymmetry breaking twist and all the supersymmetry breaking effects are contained in the vacuum expectation value of some auxiliary field. Since a vacuum expectation value of the auxiliary field can be generated dynamically through strongly coupled gauge interactions, having a small $\alpha$ parameter in the original basis is completely natural in this case. Note that this situation is quite different from the case of the $S U(5)$ breaking in the previous section, where the boundary conditions do not contain any continuous parameter and the breaking cannot be viewed as a "spontaneous breaking" that arises entirely from an expectation value of some background field [20].

We now explicitly calculate the soft supersymmetry breaking terms resulting from the boundary conditions Eqs. (12, 13). Below the compactification scale, the theory is reduced to the usual 4D MSSM. Under the KK decomposition, the MSSM gauginos $\lambda^{a}(a=1,2,3)$ are contained in the two 5D gaugino fields, $\lambda$ and $\lambda^{\prime}$, as

$$
\left(\begin{array}{c}
\lambda \\
\lambda^{\prime}
\end{array}\right)\left(x^{\mu}, y\right)=\left(\begin{array}{c}
\lambda^{a}\left(x^{\mu}\right) \cos (\alpha y / R) \\
\lambda^{a}\left(x^{\mu}\right) \sin (\alpha y / R)
\end{array}\right)+\cdots
$$

The MSSM Higgs fields $h$ arise from the two scalar fields, $\phi$ and $\phi^{c}$, in the corresponding hypermultiplets as

$$
\left(\begin{array}{c}
\phi \\
\phi^{c \dagger}
\end{array}\right)\left(x^{\mu}, y\right)=\left(\begin{array}{c}
h\left(x^{\mu}\right) \cos (\alpha y / R) \\
h\left(x^{\mu}\right) \sin (\alpha y / R)
\end{array}\right)+\cdots
$$

and similarly for the squarks and sleptons located in the bulk. The Higgsinos and the bulk quarks and leptons are zero modes of the $\psi$ fields in the corresponding hypermultiplets, and the gauge bosons come from zero modes of $A_{\mu}$. The supersymmetry breaking terms are then obtained by substituting these KK mode expansions into the original 5D action.

What supersymmetry breaking operators do we obtain? To answer this question, we first consider only the bulk interactions (kinetic terms). In our theory all the supersymmetry breaking effects are encoded in the KK mode decompositions Eqs. (14, 15), which have a $y$-dependent 
twist by the $U(1)$ subgroup of $S U(2)_{R}$. This implies that supersymmetry breaking operators arise only from the terms which contain the $y$ derivative, since the bulk Lagrangian possesses a global $S U(2)_{R}$ symmetry. The resulting supersymmetry breaking interactions are soft by dimensional analysis: the derivative $\partial_{y}$ becomes the supersymmetry breaking parameter $\alpha / R$, which has a positive mass dimension. This argument can be easily extended to the case with brane-localized interactions. The brane-localized interactions can always be made invariant under the $U(1) \subset S U(2)_{R}$; to be more precise, we can always choose brane interactions at $y=2 \pi n R(n= \pm 1, \pm 2, \cdots)$ so that the whole system is invariant under the $U(1)$ rotation by $\alpha$ and the spacetime translation $y \rightarrow y+2 \pi R$. Then, we can show that all the supersymmetry breaking terms still arise only from the terms which contain the derivative $\partial_{y}$.

The most obvious place where the $y$ derivative appears is the $5 \mathrm{D}$ kinetic terms. For the gauginos, the 5D kinetic term contains the term $-\lambda \partial_{y} \lambda^{\prime}+$ h.c., which gives Majorana mass terms for the MSSM gauginos, $-(\alpha / 2 R) \lambda^{a} \lambda^{a}+$ h.c., after integrating over $y$. The Higgs bosons obtain the soft supersymmetry breaking mass terms, $-(\alpha / R)^{2} h^{\dagger} h$, from the term $-\partial_{y} \phi^{\dagger} \partial_{y} \phi-\partial_{y} \phi^{c \dagger} \partial_{y} \phi^{c}$ in the 5D kinetic terms for the scalar fields. The same soft supersymmetry breaking mass is also induced for squarks and sleptons living in the bulk. In addition to these obvious contributions, the presence of the brane-localized Yukawa couplings also provides a somewhat non-trivial source for soft supersymmetry breaking parameters. Suppose we have a superpotential interaction $\left[\kappa \Phi_{1} \Phi_{2} \Phi_{3}\right]_{\theta^{2}}$ on the $y=0$ brane. Then, if $\Phi_{3}$ is a bulk field, we obtain the interaction term $\delta(y) \kappa \phi_{1} \phi_{2} \partial_{y} \phi_{3}^{c \dagger}+$ h.c. in the Lagrangian. (In the 4D superfield language, this term arises from eliminating the auxiliary field of $\Phi_{3}$, since the bulk kinetic term contains a superpotential term $\left[\Phi_{3} \partial_{y} \Phi_{3}^{c}\right]_{\theta^{2}}$ [21].) This term provides a trilinear scalar interaction for low energy modes $\phi_{i, 0}$ with the coefficient $\kappa \alpha / R:(\kappa \alpha / R) \phi_{1,0} \phi_{2,0} \phi_{3,0}+$ h.c.. Since exactly the same contribution is obtained from the term $\delta(y) \kappa \phi_{2} \phi_{3} \partial_{y} \phi_{1}^{c \dagger}\left(\delta(y) \kappa \phi_{3} \phi_{1} \partial_{y} \phi_{2}^{c \dagger}\right)$ if $\Phi_{1}\left(\Phi_{2}\right)$ is in the bulk, we finally obtain the trilinear scalar interaction, $\phi_{1,0} \phi_{2,0} \phi_{3,0}+$ h.c., with a coefficient given by $\kappa \alpha / R$ times the number of $\phi_{i}$ fields propagating in the bulk. Clearly, no soft supersymmetry breaking masses are generated for brane-localized fields.

To summarize, the soft supersymmetry breaking terms in our theory are given by

$$
\begin{aligned}
\mathcal{L}_{\text {soft }}= & -\frac{1}{2}\left(\tilde{m} \lambda^{a} \lambda^{a}+\text { h.c. }\right)-\tilde{m}^{2} h^{\dagger} h-\tilde{m}^{2} \tilde{f}_{B}^{\dagger} \tilde{f}_{B} \\
& +\left(y_{f} \tilde{m} \tilde{f}_{b} \tilde{f}_{b} h+2 y_{f} \tilde{m} \tilde{f}_{B} \tilde{f}_{b} h+3 y_{f} \tilde{m} \tilde{f}_{B} \tilde{f}_{B} h+\text { h.c. }\right),
\end{aligned}
$$

where $h, \tilde{f}_{B}$ and $\tilde{f}_{b}$ collectively represent the two Higgs doublets, squarks/sleptons in the bulk and squarks/sleptons on the brane, respectively. Here, we have defined $\tilde{m} \equiv \alpha / R$, and $y_{f}$ is the value of the corresponding Yukawa coupling. Since supersymmetry breaking effects from boundary conditions are exponentially shut off above the compactification scale, the soft supersymmetry breaking masses in Eq. (16) must be regarded as the running mass parameters 


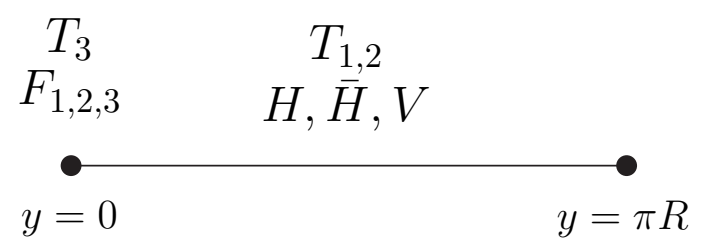

Figure 2: Locations of $S U(5)$ matter, Higgs and gauge multiplets in the fifth dimension.

at the compactification scale $M_{c}^{\prime}$. We also explicitly see that the supersymmetry breaking terms in Eq. (16) preserve the discrete $R$-parity subgroup of the $U(1)_{R}$ symmetry given in Table 2 . This is because the boundary conditions Eqs. (12, 13) always rotate two fields which differ by two units in their $U(1)_{R}$ charges. (In another basis, it comes from the fact that the auxiliary field having a supersymmetry breaking vacuum expectation value has a $U(1)_{R}$ charge of -2 .) Thus, we have $R$-parity conservation in our theory, and the lightest supersymmetric particle (LSP) is completely stable.

Now, we consider consequences of the supersymmetry breaking terms given in Eq. (16). We first note that squarks and sleptons in the bulk have non-vanishing soft masses at the compactification scale while those on the brane do not. Therefore, the brane and bulk scalars have different masses at the weak scale, even if they have the same standard model quantum numbers. This implies that the first two generation fields having the same gauge quantum number must be located in the same place to evade stringent constraints from flavor changing neutral current processes. This consideration immediately fixes the location of $T_{2}$ to be the bulk, since $T_{1}$ must be the bulk field to evade constraints from proton decay; $F_{1}$ and $F_{2}$ must also be put together either in the bulk or on the $y=0$ brane. Thus, we are finally left with only two choices for the matter location: whether we put $F_{1,2}$ in the bulk or on the brane. The locations for the other matter fields are completely fixed: $T_{3}$ and $F_{3}$ on the brane and $T_{1,2}$ in the bulk. As we will see later, the case of $F_{1,2}$ in the bulk leads to large lepton flavor violating processes, which pushes up the overall scale for supersymmetry breaking masses and leads to a fine tuning for electroweak symmetry breaking. Therefore, if we want to keep all desired features including $b / \tau$ unification and naturalness for electroweak symmetry breaking, we end up with a unique possibility for the location of matter. The resulting theory is illustrated in Fig. 2. The case without $b / \tau$ unification will be discussed in section 6 .

We here comment on the corrections to the soft supersymmetry breaking parameters at the compactification scale $M_{c}^{\prime}$. The soft parameters in Eq. (16) were derived at tree level by considering the bulk kinetic terms and the brane-localized Yukawa couplings. There are two possible sources for the corrections to these values. One comes from brane-localized kinetic 
terms for the bulk fields, and the other from finite loop radiative corrections. We first consider the effect from brane-localized kinetic terms. If there were no brane kinetic terms, the gaugino masses would be unified at $M_{c}^{\prime}$, giving gaugino mass ratios at low energies, $m_{i} / m_{j}$, different from that in the case of conventional one-scale unification; the difference amounts to more than $20 \%$ for $m_{3} / m_{1}$, leading to observable consequences. However, this does not happen due to the presence of brane-localized kinetic operators at $M_{c}^{\prime}$. After KK decomposition, the quadratic part of the MSSM gaugino Lagrangian is

$$
\mathcal{L}=\frac{1}{g_{a}^{2}} \lambda^{a \dagger} i \bar{\sigma}^{\mu} \partial_{\mu} \lambda^{a}-\frac{1}{2} \frac{\tilde{m}}{g_{*}^{2}} \lambda^{a} \lambda^{a}
$$

where $1 / g_{a}^{2}=1 / g_{*}^{2}+1 / \tilde{g}_{a}^{2}, 1 / g_{*}^{2} \equiv \pi R / g_{5}^{2}$, and $1 / \tilde{g}_{a}^{2}\left(\ll 1 / g_{*}^{2}\right)$ represent the sum of the two contributions from brane gauge kinetic terms at $y=0$ and $\pi R$. Here, all the quantities are evaluated at the compactification scale, and $g_{a}$ are the $4 \mathrm{D}$ gauge couplings at $M_{c}^{\prime}$ with effects from logarithmic running above $M_{c}^{\prime}$ included via $\tilde{g}_{a}$. Rescaling the gaugino fields to canonical normalization leads to the gaugino masses $m_{a}=\left(g_{a}^{2} / g_{*}^{2}\right) \tilde{m}$ at $M_{c}^{\prime}$, and thus $m_{a} / g_{a}^{2}$ are universal. Therefore, even though gaugino masses are generated at a smaller scale $M_{c}^{\prime}$ than the gauge coupling unification scale, we find that the grand unified relation for the gaugino masses, $m_{1} / g_{1}^{2}=m_{2} / g_{2}^{2}=m_{3} / g_{3}^{2}$, holds very precisely.

How about brane-localized kinetic terms for hypermultiplets? Since brane kinetic terms contribute to the $4 \mathrm{D}$ kinetic terms after the KK decomposition, they modify the soft masses through wavefunction renormalization. However, since effects from brane-localized terms are generically suppressed by the volume factor $M_{c}^{\prime} / M_{s}$, they only give corrections to the soft masses of order $\left(M_{c}^{\prime} / M_{s}\right) \tilde{m}^{2} \approx 10^{-2} \tilde{m}^{2}$. Although these contributions are flavor non-universal, this amount of flavor violation does not contradict with flavor changing neutral current experiments [22]; the constraints from leptonic processes, such as $\mu \rightarrow e \gamma$, are evaded for $\tilde{m} \gtrsim 200 \mathrm{GeV}$, and the bounds from hadronic ones, such as $K-\bar{K}$ mixing, are also avoided in the same parameter region partly because in the down sector only the left-handed squarks receive a flavor non-universality from the boundary conditions and partly because the gluino adds a large universal contribution through the renormalization group evolution below $M_{c}^{\prime}$. In principle, the logarithmic radiative corrections from an energy interval between $M_{s}$ and $M_{c}^{\prime}$ could enhance the contribution from the brane kinetic terms by a factor of $\ln \left(M_{s} / M_{c}^{\prime}\right)$. However, we find from dimensional analysis that Yukawa loops always give power divergences to the brane kinetic terms and thus do not enhance the contributions. The gauge loops could provide the enhancements, but they are flavor universal and thus have little observable consequences.

As for finite loop radiative corrections, they arise from non-local operators spread out in the extra dimension and appear as threshold effects at $M_{c}^{\prime}$ in a $5 \mathrm{D}$ calculation. In the $4 \mathrm{D}$ picture this corresponds to including supersymmetry breaking effects from higher KK modes. These 
contributions are shut off above $M_{c}^{\prime}$ and give only one-loop suppressed corrections, of order $1 / 16 \pi^{2} \approx 10^{-2}$, to the soft mass parameters. Thus these threshold contributions are smaller than the usual logarithmic contributions coming from renormalization group evolutions below $M_{c}^{\prime}$, by a factor of $\ln \left(M_{c}^{\prime} / M_{Z}\right)$, so that we can safely neglect them. In fact, this approximation is well justified because all the MSSM couplings are sufficiently weak at the compactification scale.

So far, we have considered only the soft supersymmetry breaking parameters arising from the kinetic terms and the Yukawa couplings. However, to understand the low energy physics, we also have to specify the Higgs sector. In particular, both the supersymmetric mass $(\mu$ parameter) and the holomorphic supersymmetry breaking mass ( $\mu B$ parameter) for the two Higgs doublets must be of the order of the weak scale to obtain viable phenomenology. In our theory these parameters can be naturally generated in a number of ways, and we will discuss some explicit examples in sub-section 5.2. However, to keep the analysis as general as possible, here we treat $\mu$ and $\mu B$ as free parameters. Then, all the supersymmetry breaking parameters in our theory are completely specified by the following four parameters: $M_{c}^{\prime}, \tilde{m}, \mu$ and $\mu B$. Among them, the last two parameters are related to the electroweak vacuum expectation value, $v \equiv\left(\left\langle h_{u}\right\rangle^{2}+\left\langle h_{d}\right\rangle^{2}\right)^{1 / 2}$, and the ratio of vacuum expectation values for the two Higgs doublets, $\tan \beta \equiv\left\langle h_{u}\right\rangle /\left\langle h_{d}\right\rangle$, through the conditions of electroweak symmetry breaking. Here $h_{u}$ and $h_{d}$ are the two Higgs doublets of the MSSM giving up-type and down-type quark masses, respectively. Since we know that $M_{c}^{\prime} \approx 10^{15} \mathrm{GeV}$ and $v \simeq 175 \mathrm{GeV}$, we are finally left with only two free parameters, $\tilde{m}$ and $\tan \beta$, to specify the superparticle spectrum. In general there is also a phase for $\mu$ which is not determined by the condition of electroweak symmetry breaking; however, if this phase is far from \pm 1 , it will lead to excessively large contributions to electric dipole moments, and hence we allow only this discrete choice. In sub-section 5.2 we give a natural origin for $\mu$ in our theory, and find that it is indeed real, solving the supersymmetric $C P$ problem. Note that various low energy quantities, including supersymmetric ones, are calculable in terms of these two parameters (and the sign of $\mu$ ) and the ratio of the cutoff and compactification scales, $M_{s} / M_{c}^{\prime}$.

Now, we present the result for the superparticle spectrum at the weak scale in our theory. The soft supersymmetry breaking parameters at $M_{Z}$ are obtained by evolving the boundary values at $M_{c}^{\prime}$, given in Eq. (16), using renormalization group equations. In Table 3, results for the soft mass parameters at $M_{Z}$ are given, in GeV, for the three MSSM gauginos, $\lambda^{a}$, for the up-type (down-type) Higgs bosons, $h_{u}\left(h_{d}\right)$, for the first two generation squarks and sleptons living in the bulk (on the brane), $\tilde{f}_{B}\left(\tilde{f}_{b}\right)$, and for the third generation squarks and sleptons, $\tilde{f}_{3}$. The parameters $A_{t}, A_{b}$ and $A_{\tau}$ are the trilinear couplings for squarks and sleptons of the third generation defined by $\mathcal{L}=-y_{f} A_{f} \tilde{f} \tilde{f} h+$ h.c.. We have given the soft masses for the 


\begin{tabular}{|cc|cc|cc|cc|cc|}
\hline$\lambda^{3}$ & 480 & $\tilde{q}_{B}$ & 480 & $\tilde{q}_{b}$ & 440 & $\tilde{q}_{3}$ & 390 & $A_{t}$ & -410 \\
$\lambda^{2}$ & 170 & $\tilde{u}_{B}$ & 470 & $\tilde{u}_{b}$ & 420 & $\tilde{u}_{3}$ & 310 & $A_{b}$ & -730 \\
$\lambda^{1}$ & 85 & $\tilde{d}_{B}$ & 470 & $\tilde{d}_{b}$ & 420 & $\tilde{d}_{3}$ & 420 & $A_{\tau}$ & -320 \\
\cline { 1 - 1 } & $280 i$ & $\tilde{l}_{B}$ & 240 & $\tilde{l}_{b}$ & 140 & $\tilde{l}_{3}$ & 140 & $|\mu|$ & 280 \\
$h_{d}$ & 240 & $\tilde{e}_{B}$ & 210 & $\tilde{e}_{b}$ & 67 & $\tilde{e}_{3}$ & 66 & $|B|$ & 95 \\
\hline
\end{tabular}

Table 3: The soft supersymmetry breaking parameters in $\mathrm{GeV}$ for $\tilde{m}=200 \mathrm{GeV}$ and $\tan \beta=5$.

first two generations of squarks and sleptons in the cases of both bulk and brane locations to maintain some generality, although these locations are completely fixed in the present model, giving $\tilde{q}_{1,2}=\tilde{q}_{B}, \tilde{u}_{1,2}=\tilde{u}_{B}, \tilde{d}_{1,2}=\tilde{d}_{b}, \tilde{l}_{1,2}=\tilde{l}_{b}$, and $\tilde{e}_{1,2}=\tilde{e}_{B}$.

In the table, we have taken the two free parameters to be $\tilde{m}=200 \mathrm{GeV}$ and $\tan \beta=5$ as a representative value. However, the dependence on $\tilde{m}$ is quite simple so that we can easily read off the soft masses for any value of $\tilde{m}$ : all the numbers scale almost linearly with $\tilde{m}$. The dependence on $\tan \beta$ is somewhat more complicated, but for moderately small values for $\tan \beta(\tan \beta \lesssim 10)$, the resulting soft masses are almost insensitive to the value of $\tan \beta$, except that $|B|$ is almost proportional to $1 / \tan \beta$. When $\tan \beta$ is further increased, several quantities vary because the bottom and tau Yukawa couplings become large. In particular, the soft mass squared for the right-handed stau becomes negative for $\tan \beta \gtrsim 25$, so that our theory does not allow very large values for $\tan \beta$.

We finally comment on several phenomenological features of the spectrum. In the MSSM the tree-level mass for the lightest $C P$ even Higgs boson is smaller than $M_{Z} \cos (2 \beta)$, so that the experimental limit requires a sizable radiative contribution. This can occur from top loop corrections to the Higgs quartic interaction [23], but, in several schemes for supersymmetry breaking, this requires the top squark to be very heavy, increasing the amount of fine tuning for successful electroweak symmetry breaking. This is not the situation for our boundary condition supersymmetry breaking because of the large $A$ parameter generated for the top quark; the predicted sign of $A$ in Eq. (16) is such that, on scaling to the infrared, $|A|$ is increased by the radiative contribution from the gaugino mass. As a result, the left-right mixing of the top squarks is large, which increases the radiative contribution to the Higgs mass. We find that for $\tan \beta=3$ (5) the Higgs mass obeys the experimental bound for $\tilde{m} \gtrsim 400(200) \mathrm{GeV}$.

Of the superpartners listed in Table 3, two have mass parameters which are significantly smaller than the rest: the right-handed scalar tau and the bino. The experimental collider signatures for our theory depend crucially on which of these particles is lighter, and yet, for several reasons, this cannot be definitively predicted. Typically we find the scalar tau to be lighter, but there are effects which could reverse the order. First, there are uncertainties in 
the mass parameters listed in Table 3. To construct a complete theory, with a natural origin for both neutrino masses and the $\mu$ parameter, in section 5 we introduce an additional gauge interaction, $U(1)_{X}(\subset S O(10) / S U(5))$, broken at some high energy scale below $M_{c}^{\prime}$ to generate Majorana masses for the right-handed neutrinos. In that case, the $U(1)_{X}$ gaugino mass leads to an additional radiative contribution to masses of the scalar superpartners which depends on the $U(1)_{X}$ breaking scale. This will give only a small percentage correction for most of the superpartners, but for the right-handed stau the correction could be sizable because it has a small mass. This alone may make the stau heavier than the bino for large values of the $U(1)_{X}$ gauge coupling. Second, there are additional effects in going from the mass parameters of Table 3 to the physical particle masses. All scalar superpartners receive corrections from electroweak symmetry breaking via the electroweak $D$ terms. Again this is most important for the scalar tau, raising its mass from $66 \mathrm{GeV}$ to $77 \mathrm{GeV}$ for $\tilde{m}=200 \mathrm{GeV}$. Hence we discuss collider phenomenology for both cases of stau and bino "LSP" . I In either case some care is required in obtaining a precise value for the lower bound on $\tilde{m}$ from experiment, but we typically expect this bound to be in the region of $200 \mathrm{GeV}$.

If the stau is lighter than the bino it will appear as a charged stable particle in collider detectors. The present limit on the mass of such a stau from the LEP experiments is about $100 \mathrm{GeV}$, coming from direct Drell-Yan production of $\tilde{\tau}^{+} \tilde{\tau}^{-}$. At hadron colliders, in addition to Drell-Yan production, stau pairs arise from squark and gluino pair production followed by cascade decays, giving equal numbers of like sign and opposite sign events. If the bino is lighter, the signal events, arising from squark and gluino pair production, contain jets and missing transverse energy — a classic signal for many supersymmetric theories. In this case the present experimental limit from LEP experiments on the stau mass is quite weak if it is close in mass to the bino.

Ultimately, an important experimental test of our theory is to measure the superpartner masses with sufficient accuracy to probe the location of the matter: at the compactification scale, all bulk superpartners have a universal mass, while all brane superpartners are massless. In the physical spectrum this is clearly manifested in the lepton sector, with $\tilde{l}$ and $\tilde{e}_{3}$ much lighter than $\tilde{e}_{1,2}$, but is harder to uncover in the squarks due to the gluino focusing effect.

\section{Consequences}

In the previous section we have explicitly demonstrated how to break both $S U(5)$ gauge symmetry and 4D supersymmetry by boundary conditions that act in the same spatial dimension.

\footnotetext{
${ }^{3}$ Our theory possesses a continuous $U(1)_{R}$ symmetry which is spontaneously broken to $R$ parity by the dynamics for an auxiliary field in the 5D supergravity multiplet, as discussed in sub-section 3.5, so that the true LSP may be the $R$ axino.
} 
This leads to a highly constrained theory with many consequences, some of which we explore in this section. The gross flavor structure of the theory is determined by the location of matter in the fifth dimension, and in the next sub-section we show how many realistic features of the quark and lepton mass matrices emerge automatically. (In the next section we explore the superpartner mass matrices, and give predictions for a variety of flavor changing phenomena.) The superpartner spectrum is so tightly constrained that we are able to evaluate the supersymmetric threshold corrections to the predictions to gauge and Yukawa coupling unification. Corrections to Yukawa unification from the compactification scale are also computed. Finally we study proton decay and the consequences of spontaneously breaking $U(1)_{R}$ symmetry.

\subsection{Quark and lepton mass matrices}

With the matter configuration determined in the previous section, quark and lepton mass matrices take the form

$$
\mathcal{L}_{4} \approx\left(\begin{array}{lll}
T_{1} & T_{2} & T_{3}
\end{array}\right)\left(\begin{array}{ccc}
\epsilon^{2} & \epsilon^{2} & \epsilon \\
\epsilon^{2} & \epsilon^{2} & \epsilon \\
\epsilon & \epsilon & \underline{1}
\end{array}\right)\left(\begin{array}{c}
T_{1} \\
T_{2} \\
T_{3}
\end{array}\right) H+\left(\begin{array}{ccc}
T_{1} & T_{2} & T_{3}
\end{array}\right)\left(\begin{array}{ccc}
\epsilon & \epsilon & \epsilon \\
\epsilon & \epsilon & \epsilon \\
\underline{1} & \underline{1} & \underline{1}
\end{array}\right)\left(\begin{array}{c}
F_{1} \\
F_{2} \\
F_{3}
\end{array}\right) \bar{H} .
$$

Here we display only the gross structure that follows from the large size of the extra dimension, via the volume suppression factor $\epsilon \simeq\left(M_{c}^{\prime} / M_{s}\right)^{1 / 2} \approx 0.1$, and suppress the coupling parameters of the brane-localized Yukawa interactions. Only underlined entries respect $S U(5)$, since the other entries involve $T_{1,2}$ which actually represent quarks and leptons from differing $S U(5)$ bulk multiplets. The only unified mass relation is for $b / \tau$.

Although the matrices of Eq. (18) do not provide a complete understanding of quark and charged lepton masses and mixings, they do capture many of the qualitative features. The masses of the first two generations are suppressed compared with the third one, by $\epsilon^{2}$ in the up quark sector and by $\epsilon$ in the down quark and charged lepton sectors. Furthermore, the quark mixing is small between the first two generations and the third. These are perhaps the most striking features of the data, and they result in our theory entirely because $T_{1,2}$ alone reside in the bulk.

The most striking prediction of these matrices is that the flavor mixing angles are of order unity for fields in $F_{i}$, which is particularly important for the neutrinos. In sub-section 5.1 we discuss how neutrino masses are generated in our theory by the see-saw mechanism, leading to the operators $[L L H H]_{\theta^{2}}$ in the low energy theory. Because $F_{i}$ all reside at the same location, we predict that the coefficients of these operators have comparable sizes for all flavor combinations. Such an anarchy can reproduce observed neutrino phenomenology [24], but is only consistent with the large angle MSW interpretation of the solar neutrino flux. 
The matrices of Eq. (18) leave two important features of the spectrum unexplained: the $t / b$ ratio and the hierarchy within the first two generations. The first requires either $\tan \beta \approx 50$ or an additional suppression of $T F \bar{H}$ relative to $T T H$. A very large value for $\tan \beta$ is not preferred, since the resulting large value for the tau Yukawa coupling leads to a scaling of $m_{\tilde{\tau}}^{2}$ to negative values at the weak scale, although this could be counteracted by a large contribution from the $U(1)_{X}$ gaugino radiative correction. Also, small values of $\tan \beta$ are excluded by the experimental limit on the mass of the lightest Higgs boson, unless the low energy theory is extended to include a singlet chiral superfield. Thus we will typically use $\tan \beta \sim 5-10$, giving a ratio of $b$ to $t$ Yukawa couplings of $\sim 1 / 5-1 / 10$.

There are at least three ways to obtain a hierarchy within the light two generations. One possibility is that there is a sufficient spread in the Yukawa coupling parameters to accommodate the data. While this is plausible for the Cabibbo angle, and perhaps also for the $d / s$ mass ratio, it seems less likely for the $u / c$ and $e / \mu$ mass ratios. A second possibility is to introduce a $U(1)$ flavor symmetry giving $T_{1}$ a different charge to $T_{2}$. For example, consider the charge assignments $T_{1}(1), T_{2,3}(0), F_{1,2,3}(1)$, where the charges for $F_{1,2,3}$ also give a suppression for the $b / t$ mass ratio. Taking the $U(1)$ symmetry breaking parameter $\delta \approx \epsilon$, we obtain a realistic pattern of masses $m_{t}: m_{c}: m_{u} \approx 1: \epsilon^{2}: \epsilon^{4}, m_{b}: m_{s}: m_{d} \approx m_{\tau}: m_{\mu}: m_{e} \approx 1: \epsilon: \epsilon^{2}$ and mixing angles $\left(V_{u s}, V_{c b}, V_{u b}\right) \approx\left(\epsilon, \epsilon, \epsilon^{2}\right)$. A third possibility is to introduce an $S_{2}$ discrete symmetry which interchanges $T_{1} \leftrightarrow T_{2}$. This forces the couplings of $T_{1}$ and $T_{2}$ to be equal, so that the second generation is $T^{+}=T_{1}+T_{2}$, while the first generation $T^{-}=T_{1}-T_{2}$ is massless in the symmetry limit. The $u$ and $d$ masses and Cabibbo angle now arise from small $S_{2}$ breaking couplings.

\subsection{Gauge coupling unification}

A theory with a supersymmetric desert and high scale gauge coupling unification leads to a prediction for the QCD coupling of the form

$$
\alpha_{s}\left(M_{Z}\right)=0.1305+\left.\delta \alpha_{s}\right|_{\text {susy }}+\left.\delta \alpha_{s}\right|_{u}
$$

The central number assumes that the $S U(5)$ split multiplets have the same form as in the MSSM [25], and should be compared with the experimental value [9]

$$
\alpha_{s}^{\exp }=0.117 \pm 0.002 .
$$

In conventional supersymmetric grand unified theories, although the prediction from gauge coupling unification is highly successful, the agreement with data is certainly not perfect. Significant threshold corrections from supersymmetric or unified thresholds are required. The weak scale threshold correction depends on the masses of the superpartners and Higgs bosons 
and has the form

$$
\left.\delta \alpha_{s}\right|_{\text {susy }} \simeq-0.0030 \sum_{r} \frac{5}{38}\left(4 b_{1}^{r}-\frac{48}{5} b_{2}^{r}+\frac{28}{5} b_{3}^{r}\right) \ln \frac{m_{r}}{M_{Z}}
$$

where the index $r$ runs over all superpartner and heavy Higgs particles, and $b_{a}^{r}$ represent the contributions of particle $r$ to the one-loop beta function coefficients [26]. Unless the supersymmetric spectrum is highly unusual, this correction is not sufficient to bring agreement with data, and hence $\left.\delta \alpha_{s}\right|_{\mathrm{u}} \neq 0$ is required. However, the threshold correction at the unified scale, $\left.\delta \alpha_{s}\right|_{\mathrm{u}}$, in general depends on parameters of the unified theory, and cannot be numerically predicted.f

In KK grand unified theories, the situation is quite different [2]. If the higher dimensional theory is valid over a sizable energy scale, up to the scale $M_{s}$ of strong coupling, then the leading unified scale corrections, coming from the KK towers, can be reliable computed. In the 5D $S U(5)$ theory the result for $\left.\delta \alpha_{s}\right|_{\mathrm{u}}$ is given in Eq. (9). Together with the finite part, calculated using dimensional regularization [28], we find

$$
\left.\delta \alpha_{s}\right|_{\mathrm{u}} ^{\mathrm{KK}} \simeq-0.0019 \ln \frac{M_{s}}{M_{c}^{\prime}}-0.0018 \simeq-0.012 \pm 0.003
$$

Here we have used $M_{s} / M_{c}^{\prime} \simeq 16 \pi^{3} / g^{2} C \simeq 200$ for our theory, which gives a corresponding error of \pm 0.003 arising from unknown physics above $M_{s}$, described in the effective theory by brane-localized operators at $M_{s}$. This contribution from KK towers precisely explains the difference between the experimental value, Eq. (20), and the central prediction without threshold corrections, Eq. (19).

Breaking supersymmetry via the small boundary condition parameter $\alpha$ allows us to go further, since we know the superpartner spectrum in terms of $\tilde{m}=\alpha / R$. Substituting the predicted masses of Table 3 into the $m_{r}$ 's of Eq. (21) gives

$$
\left.\delta \alpha_{s}\right|_{\text {susy }} ^{\mathrm{KK}} \simeq 0.0040-0.0030 \ln \frac{\tilde{m}}{M_{Z}}
$$

Here, we have approximated the masses of the heavy Higgs bosons and the Higgsinos by $\left(2|\mu|^{2}+\right.$ $\left.m_{h_{u}}^{2}+m_{h_{d}}^{2}\right)^{1 / 2}$ and $|\mu|$, respectively. Hence, in the wide parameter region $\tilde{m}=200-800 \mathrm{GeV}$, the prediction from gauge coupling unification in our theory is

$$
\alpha_{s}^{\mathrm{KK}}=(0.116 \sim 0.120) \pm 0.003
$$

This is in striking agreement with data, Eq. (20). Since threshold corrections from the weak and compactification scales have been included, as well as the logarithmic contributions from the

\footnotetext{
4 The minimal supersymmetric $S U(5)$ theory is excluded because proton stability bounds requires $\left.\delta \alpha_{s}\right|_{\mathrm{u}}$ to have the wrong sign [27].
} 
KK towers, the only significant uncertainty, \pm 0.003 , comes from unknown physics at and above the scale $M_{s}$ of strong coupling. While the general result for $5 \mathrm{D} \mathrm{KK}$ grand unified theories is highly successful, in our theory we are also able to exclude the possibility of a large correction from the supersymmetric threshold.

\subsection{Yukawa coupling unification}

Grand unified theories unify quarks with leptons and therefore have the possibility of relating quark and lepton masses. In theories where a single Yukawa coupling generates masses for both the $b$ quark and $\tau$ lepton, the mass ratio $m_{b} / m_{\tau}$ is predicted. Inputting the precisely known experimental value of the $\tau$ mass leads to a prediction for the $b$ mass

$$
m_{b}\left(M_{Z}\right)=m_{b}^{0}\left(1+\left.\frac{\delta m_{b}}{m_{b}}\right|_{\text {susy }}+\left.\frac{\delta m_{b}}{m_{b}}\right|_{\mathrm{u}}\right),
$$

which is similar in form to the prediction for $\alpha_{s}\left(M_{Z}\right)$ of Eq. (19) from gauge coupling unification, having threshold corrections from both the supersymmetry breaking and unified scales. An important difference is that the central prediction, $m_{b}^{0}$, depends on $\tan \beta$. However, in the region mainly of interest to us, $3 \lesssim \tan \beta \lesssim 20$, the dependence is weak: $m_{b}^{0}=3.6 \pm 0.1 \mathrm{GeV}$, where the uncertainty depends on both $\tan \beta$ and $\alpha_{s}$. This should be compared with the value of the running $b$ quark mass extracted from data

$$
m_{b}^{\exp }=3.0 \pm 0.3 \mathrm{GeV}
$$

Without quark-lepton unification, there is no reason to expect the $b$ and $\tau$ masses to be close, hence it is significant that the unified Yukawa coupling leads to a broadly successful prediction. Nevertheless, the prediction is too large by $(10-30) \%$. However, because the supersymmetric threshold corrections are typically large [29], one usually views this as an inherent limitation on the sensitivity of this probe of the unified theory. Finite one-loop diagrams with a virtual gluino and chargino give contributions to the bottom quark mass proportional to tan $\beta$

$$
\left.\frac{\delta m_{b}}{m_{b}}\right|_{\text {susy }} \simeq \frac{\mu \tan \beta}{4 \pi}\left[\frac{8 \alpha_{s} m_{\tilde{g}}}{3} I\left(m_{\tilde{g}}^{2}, m_{\tilde{b}_{1}}^{2}, m_{\tilde{b}_{2}}^{2}\right)+\frac{y_{t} A_{t}}{4 \pi} I\left(|\mu|^{2}, m_{\tilde{t}_{1}}^{2}, m_{\tilde{t}_{2}}^{2}\right)\right],
$$

where $m_{\tilde{g}}^{2}$ is the gluino mass, and $m_{\tilde{b}_{1,2}}\left(m_{\tilde{t}_{1,2}}\right)$ denote the masses for the two bottom (top) squarks. The function $I$ is defined by $I(a, b, c) \equiv-\{a b \ln (a / b)+b c \ln (b / c)+c a \ln (c / a)\} /\{(a-$ $b)(b-c)(c-a)\}$. Hence, in most unified theories the significance of the successful $b$ mass prediction is limited by rather poor precision. In In addition, there are threshold corrections from the unified scale, which typically cannot be calculated numerically.

\footnotetext{
${ }^{5}$ The situation is not improved at large $\tan \beta$. Although $m_{b}^{0}$ can now approach the experimental value, the contributions to the supersymmetric threshold corrections are proportional to tan $\beta$ and hence are large, unless cancellations occur in Eq. (27).
} 
The situation in our 5D $S U(5) \mathrm{KK}$ grand unified theory is quite different. As in the case of gauge coupling unification, the unified scale corrections can be computed, and the supersymmetric corrections depend on only the single parameter $\alpha$. For $m_{b}$ we are able to obtain a prediction having a smaller error bar than the current data.

First, we consider the correction from the unified scale. The largest effect in our theory arises because the bottom and tau Yukawa couplings are unified at the compactification scale $M_{c}^{\prime}$, which is smaller than the conventional unification scale $M_{u} \simeq 2 \times 10^{16} \mathrm{GeV}$. This is different from the situation in gauge coupling unification, where the couplings unify at $M_{s}$ rather than $M_{c}^{\prime}$. It is interesting to understand the different behavior of gauge and Yukawa unification. The renormalization group running below and above $M_{c}^{\prime}$ can have completely different origins in the higher dimensional picture. Below $M_{c}^{\prime}$ the running can be caused by the generation of operators which are non-local along the extra dimension, while above $M_{c}^{\prime}$ all the running must come from local operators in the extra dimension. In the case of the Yukawa couplings, the bottom and tau Yukawa couplings evolve differently below the compactification scale. This is obvious in the $4 \mathrm{D}$ picture because the effective theory below $M_{c}^{\prime}$ is just the usual MSSM, but in the 5D picture it is not so obvious, because both $T_{3}$ and $F_{3}$ are localized on the $y=0$ brane where the complete $S U(5)$ symmetry is operative. Nevertheless there is a simple understanding: below $M_{c}^{\prime}$, radiative corrections generate kinetic operators for $T_{3}$ and $F_{3}$ that are non-local along the extra dimension (effective kinetic operators involving Wilson lines or Wilson loops). Since these operators are non-local (i.e. go around the circle), they feel $S U(5)$ violating effects from the boundary conditions, and hence are $S U(5)$ violating. Clearly, the effects from these operators exponentially shut off above $M_{c}^{\prime}$ because of their non-local nature. Thus whether there is $S U(5)$ violating running at energies above $M_{c}^{\prime}$ or not is determined by whether we can write down an $S U(5)$ violating local operator or not. However, in our theory both $T_{3}$ and $F_{3}$ are localized on $S U(5)$-invariant $y=0$ brane, so their kinetic and Yukawa interactions cannot violate $S U(5)-$ above $M_{c}^{\prime}$ the bottom and tau Yukawa couplings must run together and hence are unified. This is in contrast with the case of gauge coupling unification, where the gauge fields propagate in the bulk and can have $S U(5)$ violating kinetic energy operators located on the $y=\pi R$ brane. As a result, gauge couplings have $S U(5)$ violating logarithmic evolution above $M_{c}^{\prime}$, while the third generation Yukawa couplings do not, as illustrated in Fig. 3 .

The correction from the unified scale, $\delta m_{b} /\left.m_{b}\right|_{\mathrm{u}}$, is calculated as follows. At leading order, the bottom and tau Yukawa couplings are split because they receive different contributions from QCD, hypercharge and top Yukawa radiative corrections below their unification scale. Since the unification scale in our theory, $M_{c}^{\prime}$, differs from that in conventional 4D supersymmetric 


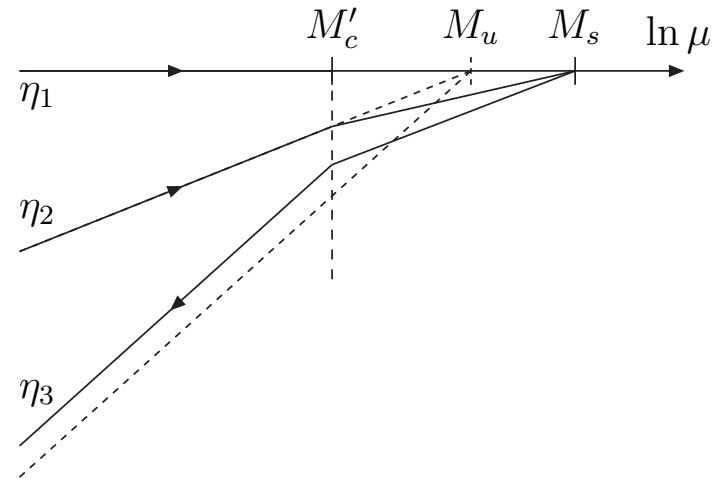

a) gauge couplings

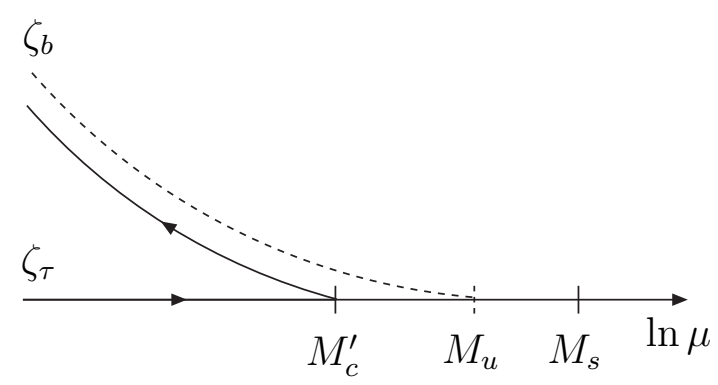

b) Yukawa couplings

Figure 3: a) The running of the difference of the three gauge couplings, $\eta_{i} \equiv \alpha_{i}^{-1}-\alpha_{1}^{-1}$. Solid and dashed lines represent the runnings in KK grand unification and conventional 4D grand unification, respectively. In KK grand unification, $\eta_{i}$ 's run logarithmically both below and above $M_{c}^{\prime}$, but with different beta function coefficients, so that the prediction for the QCD coupling differs from that in the case of a single scale unification. b) The running of the Yukawa couplings, $\zeta_{f} \equiv y_{f} / y_{\tau}-1$ in KK grand unification (solid) and 4D grand unification (dashed). In KK grand unification there is no $S U(5)$-violating running above $M_{c}^{\prime}$, so that the predicted value of the bottom quark mass is smaller than that of the single scale unification case.

unified theories, $M_{u}$, we obtain the correction

$$
\left.\frac{\delta m_{b}}{m_{b}}\right|_{\mathrm{u}} ^{\mathrm{KK}} \simeq-\frac{4 g^{2}-y_{t}^{2}}{16 \pi^{2}} \ln \frac{M_{u}}{M_{c}^{\prime}},
$$

where $g$ and $y_{t}$ represent the values of the $4 \mathrm{D}$ gauge coupling and the top Yukawa coupling at the compactification scale, $g \simeq 0.7, y_{t} \simeq 0.6$. Note that the gauge contribution dominates over the top contribution so that the net effect is to reduce the prediction for $m_{b}\left(M_{Z}\right)$ and therefore reduce the discrepancy with data. This result is independent of how supersymmetry is broken and has general applicability to all KK grand unified theories having $b / \tau$ unification. Using Eq. (10), we obtain $\delta m_{b} /\left.m_{b}\right|_{\mathrm{u}} ^{\mathrm{KK}} \simeq-\left(5\left(4 g^{2}-y_{t}^{2}\right) / 112 \pi^{2}\right) \ln \left(M_{s} / M_{c}^{\prime}\right)$, so that for our theory, $M_{s} / M_{c}^{\prime} \approx 200$, we find $\delta m_{b} /\left.m_{b}\right|_{\mathrm{u}} ^{\mathrm{KK}} \approx-4 \%$.

Substituting predictions for $\mu$ and the superpartner masses from Table 3 into the expression Eq. (27) for the supersymmetric threshold correction, we find

$$
\left.\frac{\delta m_{b}}{m_{b}}\right|_{\text {susy }} ^{\mathrm{KK}} \simeq 0.006 \times \operatorname{sgn}(\mu) \tan \beta .
$$

Strictly speaking, the mass eigenvalues for the bottom and top squarks depend on the ratio $v / \tilde{m}$; however, we have ignored this and find Eq. (29) gives a good approximate numerical 
result over a wide range of the parameter space. Choosing $\operatorname{sgn}(\mu)<0$, the prediction for the $b$ quark mass in our theory is

$$
m_{b}\left(M_{Z}\right)=3.3-0.02(\tan \beta-10) \pm 0.1 \mathrm{GeV}
$$

While a more precise form for the prediction in terms of $\tan \beta$ and $\alpha_{s}$ is possible, it is not warranted by the present error from extracting $m_{b}$ from data. The simple prediction of Eq. (30) is nevertheless quite important. Our KK theory does bring the prediction for $m_{b} / m_{\tau}$ into agreement with data without the need to invoke unknown threshold correction of at least $10 \%$. If the corrections from KK modes had gone in the wrong direction, our theory would have been very clearly excluded. Instead we predict that $m_{b}$ lies at the upper edge of the presently allowed experimental region.

\subsection{Proton decay}

In KK grand unified theories proton decay from operators of dimension four and five is forbidden [1], so that the only possible source of significant proton decay is from operators of dimension six. Since brane-localized dimension six operators, with coefficients given by inverse powers of $M_{s}$, give effects which are too small to observe, the only possible relevant source is from exchange of the superheavy gauge bosons, such as the $X$ boson of $S U(5)$. In $4 \mathrm{D}$ supersymmetric unified theories, the $X$ gauge boson exchange contribution is negligible since the $X$ gauge boson mass is $M_{X} \simeq M_{u} \simeq 2 \times 10^{16} \mathrm{GeV}$. However, in $\mathrm{KK}$ unified theories gauge coupling unification results only if the volume of the bulk is large, and this leads to lower values for $M_{X}=M_{c} / 2$, making the gauge boson exchange contribution much more important [1]. A precise prediction for gauge coupling unification results when the volume of the bulk is as large as possible, so that the gauge interactions at $M_{s}$ are strong, and this leads to low and calculable values for $M_{X}$, opening the possibility for interesting predictions for the proton decay rate [8, 2].

In fact, while gauge boson mediated proton decay is of great interest in KK grand unified theories, at present significant uncertainties remain. Exchange of $X$ gauge bosons results in baryon number violating four fermion operators of the form $\left[T_{i}^{\dagger} T_{i} T_{j}^{\dagger} T_{j}\right]_{\theta^{2} \bar{\theta}^{2}}$ and $\left[T_{i}^{\dagger} T_{i} F_{j}^{\dagger} F_{j}\right]_{\theta^{2} \bar{\theta}^{2}}$. The baryon number violating interactions amongst zero modes are generated only when $T$ and $F$ are brane fields, since for bulk fields the $X$ boson couples a zero mode to a superheavy state. The coefficients for these operators are given by $\sum_{n} g_{X}^{2} /\left((n+1 / 2) M_{c}\right)^{2}=g^{2} / M_{c}^{\prime 2}$, where $g_{X}$ and $g$ are the gauge couplings for the $X$ and standard model gauge bosons, respectively, and

\footnotetext{
${ }^{6}$ In the case where small neutrino masses are generated through the see-saw mechanism, the presence of Dirac neutrino Yukawa couplings at high energies could increase the prediction for $m_{b}$ by a small amount but does not change our conclusions. The $U(1)_{X}$ gauge interaction does not renormalize $m_{b} / m_{\tau}$ and hence does not affect our prediction.
} 
$g_{X}=\sqrt{2} g$ due to the wavefunction profiles of the gauge fields in the extra dimension. Since the corresponding coefficients are given by $g_{X}^{2} / M_{X}^{2}=g^{2} / M_{X}^{2}$ in $4 \mathrm{D}$ grand unified theories, $M_{c}^{\prime}$ in 5D theories corresponds to $M_{X}$ in $4 \mathrm{D}$ theories as far as dimension six proton decay is concerned. For $M_{c}^{\prime} \approx 10^{15} \mathrm{GeV}$, present limits on proton decay require that $T_{1}$ be a bulk field. The bulk gauge interactions then generate proton decay through quark mass mixing and/or brane-localized interactions [8, 30]. This introduces uncertainties for the proton decay rate, since the relevant mixing matrix is not the CKM matrix of weak interactions and the size of brane interactions are not determined by gauge coupling unification. Nevertheless, it is still interesting to pursue the natural expectation for the rate for various proton decay modes in these KK grand unified theories.

In our theory $F_{i}$ are all brane fields, but this is not sufficient to generate baryon number violation. Since only the third generation is on the brane for $T$ fields, proton decay is dominated by the operator $\left[T_{3}^{\dagger} T_{3} F_{1}^{\dagger} F_{1}\right]_{\theta^{2} \bar{\theta}^{2}}$ and hence requires mixing via the third generation of quarks. We then find that the four fermion interaction $\mathcal{L} \supset\left(g^{2} / M_{c}^{\prime 2}\right)\left(V_{23}^{q \dagger} V_{31}^{u}\right) q_{2}^{\dagger} u_{1} l_{1}^{\dagger} d_{1}$ gives the dominant proton decay amplitude, where $V^{q, u}$ is the unitary rotation on $q, u$ needed to diagonalize the quark mass matrices. Since we must take the strange quark from $q_{2}$ to obtain a proton decay amplitude, $V^{q}$ is actually the rotation in the left-handed down quark sector and the final states for the decay contain the particle carrying the strangeness quantum number. This leads to the decay mode $p \rightarrow K^{+} \bar{\nu}$ with the lifetime estimate $\tau\left(p \rightarrow K^{+} \bar{\nu}\right) \approx 10^{37 \pm 2}$ years. Here, we have arbitrarily assigned one order of magnitude uncertainty to $M_{c}^{\prime 4}$ and one to the flavor mixing angles; the central value comes from taking $V_{23}^{q \dagger} V_{31}^{u} \simeq 0.0002$ and $M_{c}^{\prime}=M_{u}\left(M_{c}^{\prime} / M_{s}\right)^{5 / 7}$ with $M_{s} / M_{c}^{\prime} \simeq 16 \pi^{3} / g^{2} C \simeq 200$. The simple expectation is therefore that this flavor mixing contribution to proton decay is too small to observe. In general in KK unified theories one only expects an observable rate if $T_{2}$ is on the brane [8, 2], or if $M_{c}^{\prime}$ is of order $10^{14} \mathrm{GeV}$ rather than of order $10^{15} \mathrm{GeV} 30$.

Symmetries of our theory allow the operators $\left[\left(\bar{g}_{i j} / M_{s}\right) T_{i}^{\dagger} T_{j}^{\prime}\right]_{\theta^{2} \bar{\theta}^{2}}$ on the $y=0$ brane. After the KK mode decomposition, these operators give baryon number violating couplings amongst zero modes: $\mathcal{L} \supset \bar{g}_{i j}\left(M_{c}^{\prime} / M_{s}\right) u_{i}^{\dagger} \gamma^{\mu} q_{j} X_{\mu}$. The leading proton decay in our theory comes from combining these interactions with the $d_{i}^{\dagger} \gamma^{\mu} l_{i} X_{\mu}$ interactions coming from the usual kinetic terms $\left[F_{i}^{\dagger} F_{i}\right]_{\theta^{2} \bar{\theta}^{2}}$ on the brane. The resulting decay amplitudes are independent of the Yukawa interactions (i.e. quark mixing matrices), but depend on the unknown coefficients $\bar{g}_{i j}$. We find that $\bar{g}_{22}$ and $\bar{g}_{21}$ do not lead to proton decay amplitudes at leading order in $\left(M_{c}^{\prime} / M_{s}\right)$, but $\bar{g}_{12}$ and $\bar{g}_{11}$ lead to $p \rightarrow K^{+} \bar{\nu}$ and $p \rightarrow e^{+} \pi^{0}, \mu^{+} \pi^{0}, e^{+} K^{0}, \mu^{+} K^{0}, \pi^{+} \bar{\nu}, K^{+} \bar{\nu}$ decay modes, respectively, both at order $\left(M_{c}^{\prime} / M_{s}\right)$ in the amplitudes. A crucial question is whether the origin of the $d / s, u / c$ ratios and the Cabibbo angle leads to further suppression of $\bar{g}_{11,12}$ relative to $\bar{g}_{22}$. In any of the examples given in sub-section 3.1, there is no such suppression for $\bar{g}_{11}$, 
so we expect the coefficient $\bar{g}_{11}$ to be of order one. This gives the "effective $X$ gauge boson mass" $M_{X}^{\mathrm{eff}} \simeq \bar{g}_{11}^{-1 / 2}\left(M_{c}^{\prime} / M_{s}\right)^{3 / 14} M_{u}$, leading to the lifetime estimate $\tau(p) \approx 10^{34}$ years for all the decay modes $e^{+} \pi^{0}, \mu^{+} \pi^{0}, e^{+} K^{0}, \mu^{+} K^{0}, \pi^{+} \bar{\nu}$ and $K^{+} \bar{\nu}$, for $\bar{g}_{11} \simeq 1$ and $M_{s} / M_{c}^{\prime} \simeq 200$. An interesting point is that, although the total proton decay rate has uncertainties coming from $\bar{g}_{11}$, the relative decay rates for various decay modes are predicted essentially in terms of a single unknown parameter $\theta_{F}$. This is because we can always choose a gauge eigenbasis for $F_{i}$ so that only $F_{3}$ couples to $T_{3}$, and in that basis the only large mixing angle for quarks and leptons in $F_{i}$ is that between the first two generations (see also the discussion around Eq. (34)). We define the relative mixing angles between the down type quarks and the lepton doublets as $\theta_{F}$, which we expect to be of order unity. We then obtain a number of predictions for the relative decay rates, so that the physics of proton decay is very rich in our theory. Particularly useful relations are

$$
\frac{\Gamma\left(p \rightarrow \mu^{+} \pi^{0}\right)}{\Gamma\left(p \rightarrow e^{+} \pi^{0}\right)} \simeq \frac{\Gamma\left(p \rightarrow e^{+} K^{0}\right)}{\Gamma\left(p \rightarrow \mu^{+} K^{0}\right)} \simeq \tan ^{2} \theta_{F} .
$$

This is a robust prediction because it does not depend on hadronic matrix elements nor whether $\bar{g}_{12}$ is sizable. For the first discovery, $e^{+} \pi^{0}$ will be the most promising mode for an experimental search, since it has a relatively clean signature. We stress that, while our analysis for proton decay depends on matter location, it is completely independent of supersymmetry breaking.

\subsection{Axion and axino from $U(1)_{R}$}

The bulk interactions of our theory possess an $S U(2)_{R} \times S U(2)_{H}$ global symmetry, and we have argued that it is crucial for the $U(1)_{R}$ subgroup to be preserved also by all the brane interactions. On the other hand the small boundary condition parameter $\alpha$ clearly breaks this $U(1)_{R}$ symmetry. However, this breaking will ultimately be spontaneous, arising from the vacuum expectation value of an auxiliary field in the $5 \mathrm{D}$ supergravity multiplet. It is therefore natural to explore the possibility that the global $U(1)_{R}$ symmetry is an exact symmetry of the entire theory, except for gauge anomalies, so that an $R$ axion arises from the spontaneous breaking of $U(1)_{R}$. An interesting possibility is that the $R$ axion only receives mass from the gauge interactions of the standard model. (In fact, in section 5 we will introduce an additional gauge interaction which sets the $U(1)_{X}$ breaking scale, but $U(1)_{R}$ does not have an anomaly for this gauge group so that the $R$ axion does not get a mass from this gauge interaction.) If this is the case, the $R$ axion receives the dominant mass contribution from the QCD anomaly of $U(1)_{R}$ and thus plays the role of the QCD axion, solving the strong $C P$ problem [31]. The $U(1)_{R}$ breaking scale is given (if these is no other $U(1)_{R}$ breaking) by the supersymmetry breaking 
vacuum expectation value of the auxiliary field: $F_{a} \approx\left(\tilde{m} M_{\mathrm{Pl}}\right)^{1 / 2} \approx 10^{10}-10^{11} \mathrm{GeV}$.

We here discuss possible phenomenological consequences of this exact $U(1)_{R}$ scenario. First, the mass of the (fermionic) superpartner of the $R$ axion, the $R$ axino, strongly depends on the sector which breaks $U(1)_{R}$ spontaneously. In particular, the $R$ axino could be much lighter than all the superpartners of the standard model particles. In this case, the lightest standard model superpartner can decay into the $R$ axino, so that there is no problem of charged dark matter even if the stau is the lightest of the MSSM superparticles. The lifetimes for stau and neutralino decays are estimated roughly to be $10^{3}\left(F_{a} / 10^{11} \mathrm{GeV}\right)^{2}\left(10^{2} \mathrm{GeV} / m_{\tilde{\tau}}\right)^{3}$ sec and $10^{-3}\left(F_{a} / 10^{11} \mathrm{GeV}\right)^{2}\left(10^{2} \mathrm{GeV} / m_{\tilde{\chi}}\right)^{3}$ sec, respectively. The dark matter may be provided by the $R$ axion or by the $R$ axino itself.

\section{Supersymmetric Flavor Violation}

In this section we propose lepton flavor violation as a powerful and generic signal for KK grand unification providing the scale of mediation for supersymmetry breaking is at or above the compactification scale. We first consider the general consequences for flavor symmetry imposed by matter locality and experimental limits on lepton flavor violation. We then apply this to our model and derive rates for various signals in the lepton sector. We argue that hadronic signals are likely to be harder to detect.

\subsection{Flavor symmetry in Kaluza-Klein grand unification}

In supersymmetric theories, flavor violation provides an important probe of the high energy theory via the form of the soft supersymmetry breaking interactions [32]. Furthermore, the form of the soft operators reflects the underlying flavor symmetry of the theory. For example, in conventional 4D $S U(5)$ grand unified theories, the gauge interactions possess a $U(3)_{T} \times$ $U(3)_{F}$ global flavor symmetry. If soft supersymmetry breaking operators are local up to the unification scale, in the flavor symmetry limit the squark and slepton mass matrices have the form $m_{\tilde{T}}^{2}\left(\tilde{q} \tilde{q}^{\dagger}+\tilde{u}^{\dagger} \tilde{u}+\tilde{e} \tilde{e}^{\dagger}\right)+m_{\tilde{F}}^{2}\left(\tilde{d}^{\dagger} \tilde{d}+\tilde{l}^{\dagger} \tilde{l}\right)$. Here $\tilde{q}, \tilde{e}(\tilde{u}, \tilde{d}, \tilde{l})$ are three dimensional row (column) vectors in flavor space. Flavor violating signals only arise from the breaking of flavor symmetry due to non-gauge interactions, such as the top quark Yukawa coupling.

On the other hand, in KK grand unified theories the flavor symmetry of the gauge interactions depends on the location of the matter. In 5D theories with a large radius of the extra dimension, we have seen that $T_{1}$ and $T_{3}$ have differing locations, so that $U(3)_{T}$ is certainly broken. If soft supersymmetry breaking operators are generated at or above the compactification

7 If a constant term in the superpotential canceling the cosmological constant is generated by a vacuum expectation value as $W=\langle\Phi\rangle^{3}$, then the scale of $U(1)_{R}$ breaking is given by $F_{a} \approx\left(\tilde{m} M_{\mathrm{Pl}}^{2}\right)^{1 / 3} \approx 10^{13} \mathrm{GeV}$. 
scale this implies a large mass splitting between the scalars in $T_{1}$ and those in $T_{3}$. However, constraints from flavor violating processes place powerful limits on the degree to which $U(3)_{T} \times U(3)_{F}$ can be "broken" by locality. For example, $T_{1}$ and $T_{2}$ must have a common location, as must $F_{1,2,3}$, to avoid too large lepton flavor violation, as will be seen later. Hence in KK grand unified theories with a "large" extra dimension we find that the most general form for the flavor symmetry is $(U(2) \times U(1))_{U} \times(U(2) \times U(1))_{E} \times(U(2) \times U(1))_{Q} \times U(3)_{D} \times U(3)_{L}$, leading to the soft scalar masses

$$
-\mathcal{L}=m_{\tilde{q}}^{2} \tilde{q} P_{q} \tilde{q}^{\dagger}+m_{\tilde{u}}^{2} \tilde{u}^{\dagger} P_{u} \tilde{u}+m_{\tilde{d}}^{2} \tilde{d}^{\dagger} \tilde{d}+m_{\tilde{l}}^{2} \tilde{l}^{\dagger} \tilde{l}+m_{\tilde{e}}^{2} \tilde{e} P_{e} \tilde{e}^{\dagger}
$$

where $P_{q, u, e}$ are $3 \times 3$ matrices $P_{q, u, e}=\operatorname{diag}\left(1,1, c_{q, u, e}\right)$ containing arbitrary parameters $\left.c_{q, u, e} \cdot\right]^{[}$ Therefore, unless $c_{q}=c_{u}=c_{e}=1$, the soft supersymmetry breaking masses are not flavor universal even in the "flavor symmetric" limit. Here, by allowing $m_{\tilde{q}}^{2}, m_{\tilde{u}}^{2}, m_{\tilde{d}}^{2}, m_{\tilde{l}}^{2}, m_{\tilde{e}}^{2}$ (and $\left.c_{q}, c_{u}, c_{e}\right)$ to be independent, we have allowed for the case that supersymmetry breaking directly feels the breaking of gauge symmetry. This can occur easily in KK unified theories since there are locations in the bulk which have explicit breaking of the unified symmetry. The three standard model gaugino masses must also be treated as independent parameters in general in KK grand unified theories. In the case that supersymmetry breaking respects the $S U(5)$ symmetry, the flavor symmetry is reduced to $U(1)_{T_{3}} \times U(2)_{(U, E)_{1,2}} \times U(2)_{Q_{1,2}} \times U(3)_{D} \times U(3)_{L}$ and Eq. (32) holds with $m_{\tilde{u}}^{2}=m_{\tilde{e}}^{2}, c_{u}=c_{e}$ and $m_{\tilde{q}}^{2} c_{q}=m_{\tilde{u}}^{2} c_{u}$. The gaugino masses respect the unified mass relations. This is the case in our boundary condition supersymmetry breaking. Much of the remaining parameter freedom then arises because bulk matter is not fully unified; for instance, if $F_{i}$ are brane fields then we also have $m_{\tilde{d}}^{2}=m_{\tilde{l}}^{2}$. However, these additional restrictions on parameters do not eliminate flavor non-universalities, since we still have parameters $c_{q}$ and $c_{e}$, breaking flavor universality. Hence, from flavor symmetry grounds alone we see that KK grand unified theories with supersymmetry breaking mediated at or above the compactification scale generically have large lepton flavor violation originating from the split in location between $\tilde{e}_{3}$ and $\tilde{e}_{1,2}$. Below we calculate the rates in our theory — but the signal is generic to a wide class of KK unified theories with high mediation scales. In fact, the signal does not require grand unification: a separation of lepton locations is sufficient. In the unified case this separation is required, but even in non-unified theories such a separation may well contribute to fermion mass hierarchies. If supersymmetry breaking is mediated at scales well beneath the compactification scale, $U(3)^{5}$ flavor symmetry can emerge as an accidental low energy symmetry despite its short distance breaking by matter locality, removing the signal.

The flavor symmetry in our theory is $U(1)_{T_{3}} \times U(2)_{T_{1,2}} \times U(2)_{T_{1,2}^{\prime}} \times U(3)_{F}$, due to the unique choice for the location of matter, shown in Fig. 2. This flavor symmetry determines the

${ }^{8}$ If $c_{e}$ happens to be close to unity, $F_{1,2}$ and $F_{3}$ could have different locations and the flavor symmetry could be $(U(2) \times U(1))^{5}$. 
overall flavor structure of the theory, especially that of supersymmetry breaking parameters. To understand the consequences of this flavor symmetry, we first consider the limit where all the Yukawa couplings are set equal to zero. Boundary condition breaking of supersymmetry by the parameter $\alpha$ generates non-holomorphic scalar masses for squarks, sleptons and Higgs bosons. Since the boundary condition breaking does not introduce any additional flavor symmetry breaking, these supersymmetry breaking operators must respect the flavor symmetry of the gauge sector, giving soft masses of the form Eq. (32) with $m_{\tilde{u}}^{2}=m_{\tilde{e}}^{2}, c_{u}=c_{e}, m_{\tilde{q}}^{2} c_{q}=m_{\tilde{u}}^{2} c_{u}$ and $m_{\tilde{d}}^{2}=m_{\tilde{l}}^{2}$. Thus, if $c_{q}$ or $c_{e} \neq 1$, the soft supersymmetry breaking masses are not flavor universal even in the limit of vanishing Yukawa couplings. In fact, in the present theory, $m_{\tilde{q}}^{2}=m_{\tilde{u}}^{2}=m_{\tilde{e}}^{2}=\tilde{m}^{2}, m_{\tilde{d}}^{2}=m_{\tilde{l}}^{2}=0$ and $c_{q}=c_{u}=c_{e}=0$ at the compactification scale, and thus there are flavor non-universalities already at this level. This situation is quite different from that of conventional 4D unified theories where all the flavor non-universalities come from renormalization effects through the Yukawa couplings.

We next turn to the Yukawa couplings. The flavor symmetries of the 5D gauge interactions are broken by the brane-localized Yukawa couplings of Eq. (11). Flavor hierarchies for quark and lepton masses and mixings arise from geometrical volume suppressions, as shown in Eq. (18), explaining much of the gross structure of these hierarchies. In the low energy theory below the compactification scale, this flavor symmetry breaking is represented by the three superpotential Yukawa interactions

$$
W=Q \mathbf{y}_{\mathbf{U}} U H_{u}-Q \mathbf{y}_{\mathbf{D}} D H_{d}-E \mathbf{y}_{\mathbf{E}} L H_{d}
$$

where $\mathbf{y}_{\mathbf{U}}\left(\mathbf{y}_{\mathbf{D}, \mathbf{E}}\right)$ is a $3 \times 3$ matrix and has roughly the form of $y_{T}\left(y_{F}\right)$ shown in Eq. (18); $Q, E$ $(U, D, L)$ are three dimensional row (column) vectors in the flavor space. Since the soft scalar masses for $F_{i}$ (i.e. $D_{i}$ and $L_{i}$ ) are flavor universal, we can choose a basis for $F_{i}$ so that only $F_{3}$ couples to $T_{3}$ by rotating full supermultiplets $F_{i}$, giving

$$
\mathbf{y}_{\mathbf{D}, \mathbf{E}} \approx\left(\begin{array}{ccc}
\epsilon & \epsilon & \epsilon \\
\epsilon & \epsilon & \epsilon \\
0 & 0 & 1
\end{array}\right) .
$$

This shows that there is no large mixing effect in the charged lepton sector, in spite of the presence of apparent large mixing angles in Eq. (18). This remains true no matter which mechanism is used to provide a hierarchy between the first two generations. The large mixing angles appear in physical processes only when we consider the masses for neutrinos; in particular, they appear as large mixing angles in neutrino oscillation phenomena.

To summarize, the soft supersymmetry breaking operators in our theory involve a flavor 
non-universality matrix $P_{T}$ associated with the states $Q, U, E$ of $T$ :

$$
P_{T}=\left(\begin{array}{lll}
1 & 0 & 0 \\
0 & 1 & 0 \\
0 & 0 & 0
\end{array}\right) .
$$

The flavor universality is also broken by the Yukawa couplings, which we can view as flavor symmetry breaking spurions. Applying the general result Eq. (16) for the soft operators to our theory, we find the following soft terms for squarks and sleptons:9

$$
\begin{aligned}
\mathcal{L}_{\text {soft }}= & \tilde{m}\left(\tilde{q}\left(\mathbf{y}_{\mathbf{U}}+P_{T} \mathbf{y}_{\mathbf{U}}+\mathbf{y}_{\mathbf{U}} P_{T}\right) \tilde{u} h_{u}-\tilde{q}\left(\mathbf{y}_{\mathbf{D}}+P_{T} \mathbf{y}_{\mathbf{D}}\right) \tilde{d} h_{d}-\tilde{e}\left(\mathbf{y}_{\mathbf{E}}+P_{T} \mathbf{y}_{\mathbf{E}}\right) \tilde{l} h_{d}\right) \\
& -\tilde{m}^{2}\left(\tilde{q} P_{T} \tilde{q}^{\dagger}+\tilde{u}^{\dagger} P_{T} \tilde{u}+\tilde{e} P_{T} \tilde{e}^{\dagger}\right) .
\end{aligned}
$$

This tree-level result corresponds to the initial condition at the compactification scale for the renormalization group scaling of the soft parameters of the MSSM.

\subsection{Lepton flavor violation}

\subsubsection{A single lepton flavor mixing matrix}

To study the experimental consequences of lepton flavor violation in our theory, we must first scale the operators $\left[E L H_{d}\right]_{\theta^{2}}, \tilde{e} \tilde{l} h_{d}, \tilde{l}^{\dagger} \tilde{l}$ and $\tilde{e} \tilde{e}^{\dagger}$ to the weak scale. In the case that $\tan \beta$ is not too large, so that radiative effects from $y_{b, \tau}$ can be ignored, the only important scalings are due to the electroweak gauge interactions. Thus the overall form of the Yukawa matrix $\mathbf{y}_{\mathbf{E}}$ is unchanged and has the form Eq. (34) also at the weak scale. There is a radiative correction $\delta A$ to the trilinear scalar operator proportional to the gaugino mass, and there are gauge radiative corrections to the soft scalar mass parameters, giving

$$
\begin{aligned}
\mathcal{L}_{\mathrm{soft}}^{\text {lept }} \simeq- & -\tilde{e}\left[\left(\begin{array}{ccc}
2 \tilde{m}+\delta A & 0 & 0 \\
0 & 2 \tilde{m}+\delta A & 0 \\
0 & 0 & \tilde{m}+\delta A
\end{array}\right) \mathbf{y}_{\mathbf{E}}\right] \tilde{l} h_{d}+\text { h.c. } \\
& -\tilde{e}\left(\begin{array}{ccc}
m_{\tilde{e}}^{2} & 0 & 0 \\
0 & m_{\tilde{e}}^{2} & 0 \\
0 & 0 & m_{\tilde{\tau}}^{2}
\end{array}\right) \tilde{e}^{\dagger}-\tilde{l}^{\dagger}\left(\begin{array}{ccc}
m_{\tilde{l}}^{2} & 0 & 0 \\
0 & m_{\tilde{l}}^{2} & 0 \\
0 & 0 & m_{\tilde{l}}^{2}
\end{array}\right) \tilde{l},
\end{aligned}
$$

where, using Table 3, $m_{\tilde{e}}^{2}=m_{\tilde{e}_{B}}^{2} \simeq(1.1 \tilde{m})^{2}, m_{\tilde{\tau}}^{2}=m_{\tilde{e}_{3}}^{2} \simeq(0.33 \tilde{m})^{2}, m_{\tilde{l}}^{2}=m_{\tilde{l}_{b}}^{2} \simeq m_{\tilde{l}_{3}}^{2} \simeq$ $(0.70 \tilde{m})^{2}$ and $\delta A=0.60 \tilde{m}$.

By rotating to a mass eigenstate basis for charged leptons, while maintaining diagonal scalar mass-squared matrices, we can go to a basis where the lepton flavor violation appears only via a

\footnotetext{
${ }^{9}$ The most general form of the trilinear scalar interactions consistent with the flavor symmetry of the $5 \mathrm{D}$ gauge interactions is $-\mathcal{L}=\tilde{q}\left(A_{u} \mathbf{y}_{\mathbf{U}}+A_{u}^{\prime} P_{q} \mathbf{y}_{\mathbf{U}}+A_{u}^{\prime \prime} \mathbf{y}_{\mathbf{U}} P_{u}\right) \tilde{u} h_{u}-\tilde{q}\left(A_{d} \mathbf{y}_{\mathbf{D}}+A_{d}^{\prime} P_{q} \mathbf{y}_{\mathbf{D}}\right) \tilde{d} h_{d}-\tilde{e}\left(A_{e} \mathbf{y}_{\mathbf{E}}+A_{e}^{\prime} P_{e} \mathbf{y}_{\mathbf{E}}\right) \tilde{l} h_{d}$.
} 
single new mixing matrix $W^{e}$ in the following lepton-slepton-gaugino, slepton-lepton-Higgsino and slepton-slepton-Higgs interactions:

$$
\begin{aligned}
\mathcal{L}^{\mathrm{LFV}}= & -\left(\sqrt{2} g^{\prime} e W^{e \dagger} \tilde{e}^{\dagger} \tilde{b}+\text { h.c. }\right)+\left(\tilde{e} W^{e} \hat{\mathbf{y}}_{\mathbf{E}} l \tilde{h}_{d}+\text { h.c. }\right) \\
& -\left(\tilde{e}\left((\tilde{m}+\delta A) I+\tilde{m} P_{T}\right) W^{e} \hat{\mathbf{y}}_{\mathbf{E}} \tilde{l} h_{d}-\mu \tilde{e} W^{e} \hat{\mathbf{y}}_{\mathbf{E}} h_{u}^{\dagger} \tilde{l}+\text { h.c. }\right),
\end{aligned}
$$

where $\hat{\mathbf{y}}_{\mathbf{E}}$ is the real and diagonal lepton Yukawa matrix after the rotation, and $I$ is the unit $3 \times 3$ matrix; $\tilde{b}$ represents the $U(1)_{Y}$ gaugino. Note that, because the mass-squared matrix for $\tilde{l}$ is proportional to the unit matrix, the lepton flavor violation is associated with $\tilde{e}$ rather than with $\tilde{l}$.⿴囗十 The degeneracy of $\tilde{e}_{1,2}$ allows $W^{e}$ to depend on only two physical Euler angles and two phases as $W^{e}=R_{23}^{e} R_{12}^{e} D$, where $R_{i j}^{e}$ is a matrix rotating the $i j$ plane in flavor space, and $D$ is a diagonal phase matrix with two independent phases. A simultaneous phase rotation for $l, \tilde{l}$ and $e^{\dagger}$ (i.e. $l \rightarrow D^{\dagger} l, \tilde{l} \rightarrow D^{\dagger} \tilde{l}$ and $e^{\dagger} \rightarrow D^{\dagger} e^{\dagger}$ ) can further remove the phase matrix $D$ from $W^{e}$ without affecting other interactions, so that we finally obtain

$$
W^{e}=R_{23}^{e} R_{12}^{e}=\left(\begin{array}{ccc}
c_{12}^{e} & -s_{12}^{e} & 0 \\
s_{12}^{e} c_{23}^{e} & c_{12}^{e} c_{23}^{e} & -s_{23}^{e} \\
s_{12}^{e} s_{23}^{e} & c_{12}^{e} s_{23}^{e} & c_{23}^{e}
\end{array}\right)
$$

where $c_{i j}^{e} \equiv \cos \left(\theta_{i j}^{e}\right)$ and $s_{i j}^{e} \equiv \sin \left(\theta_{i j}^{e}\right)$. Therefore, we find a remarkable result that all the lepton flavor violating processes are completely described by two angles, $\theta_{12}^{e}$ and $\theta_{23}^{e}$, as far as the charged lepton sector is concerned.

\subsubsection{Branching ratio for $\mu \rightarrow e \gamma$}

In this sub-section we consider $\mu \rightarrow e \gamma$ decay. This process arises from the one-loop diagrams of Fig. 1, where sleptons and neutralinos circulate in the loop. We first consider the two diagrams of Fig. 4 $4 \mathrm{a}$ and Fig. Gb, in which both vertices arise from the $U(1)_{Y}$ gauge coupling. In this case, the decay rate for $\mu \rightarrow e \gamma$ is given by

$$
\Gamma(\mu \rightarrow e \gamma)=\frac{\alpha}{4} m_{\mu}^{3}\left|F^{(a)}+F^{(b)}\right|^{2}
$$

where $\alpha$ and $m_{\mu}$ represent the fine structure constant and the muon mass, respectively. Here, the amplitudes $F^{(a)}$ and $F^{(b)}$ are given by

$$
\begin{aligned}
F^{(a)}= & \frac{\alpha}{4 \pi \cos ^{2} \theta_{w}} m_{\mu} W_{\tau \mu}^{e} W_{\tau e}^{e *}\left[G_{1}\left(m_{\tilde{\tau}}^{2}\right)-G_{1}\left(m_{\tilde{e}}^{2}\right)\right], \\
F^{(b)}= & -\frac{\alpha}{4 \pi \cos ^{2} \theta_{w}} m_{\mu} W_{\tau \mu}^{e} W_{\tau e}^{e *} \\
& \times\left[(2 \tilde{m}+\delta A+\mu \tan \beta)\left\{G_{2}\left(m_{\tilde{l}}^{2}, m_{\tilde{\tau}}^{2}\right)-G_{2}\left(m_{\tilde{l}}^{2}, m_{\tilde{e}}^{2}\right)\right\}-\tilde{m} G_{2}\left(m_{\tilde{l}}^{2}, m_{\tilde{\tau}}^{2}\right)\right],
\end{aligned}
$$

\footnotetext{
10 This is similar to lepton flavor violation in $4 \mathrm{D}$ supersymmetric $S U(5)$ theories with $\tilde{e}$ non-degeneracy arising from radiative corrections involving the large coupling $y_{T}$ for the third generation [33].
} 


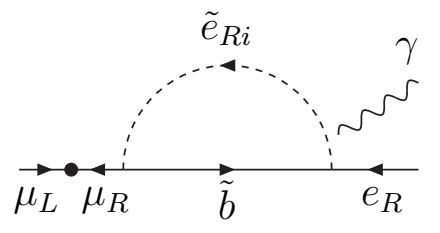

(a)

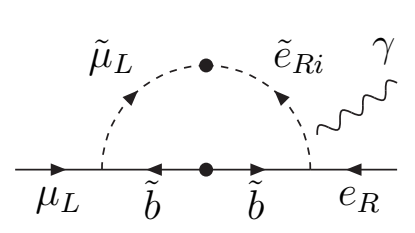

(b)

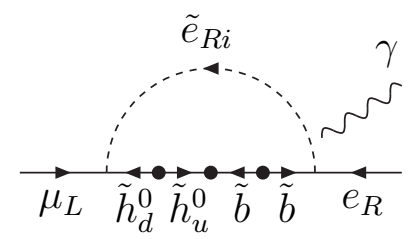

(c)

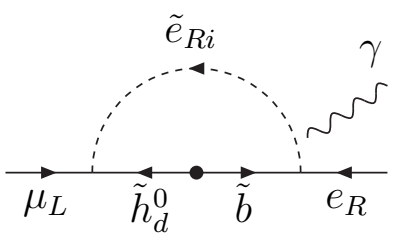

(d)

Figure 4: Feynman diagrams contributing to the $\mu \rightarrow e \gamma$ process. The chirality flips represented by blobs come from the muon mass, the gaugino and Higgsino masses, the left-right slepton mixing, and gaugino-Higgsino mixings.

where $\theta_{w}$ is the Weinberg angle, and the functions $G_{1}$ and $G_{2}$ are defined in Ref. [34. Substituting the predictions of the superparticle mass spectrum in our theory, these amplitudes are written in terms of a single mass scale $\tilde{m}$ as

$$
F^{(a)}+F^{(b)} \simeq(0.2+0.8 \operatorname{sgn}(\mu) \tan \beta) \frac{\alpha}{4 \pi \cos ^{2} \theta_{w}} \frac{m_{\mu}}{\tilde{m}^{2}} W_{\tau \mu}^{e} W_{\tau e}^{e *} .
$$

We find that in the parameter region of our interest, $\tan \beta \gtrsim 3$, the $\mu \rightarrow e \gamma$ process is dominated by the contribution proportional to $\tan \beta$, which comes from the diagram of Fig. 估b.

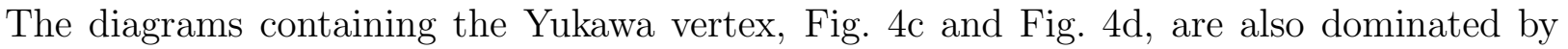

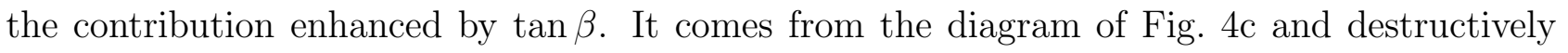
interferes with the previous contribution in Eq. (43) [35]. Using our superparticle spectrum, we find $F^{(c)} \simeq-0.4 F^{(b)}$, which reduces the rate of $\mu \rightarrow e \gamma$ by about a factor of 3 compared with that given by Eq. (43). Therefore, the $\mu \rightarrow e \gamma$ decay rate in our theory is given by

$$
\Gamma(\mu \rightarrow e \gamma) \simeq \frac{c_{\mu}^{2} \alpha^{3}}{64 \pi^{2} \cos ^{4} \theta_{w}} \frac{m_{\mu}^{5}}{\tilde{m}^{4}}\left|W_{\tau \mu}^{e}\right|^{2}\left|W_{\tau e}^{e}\right|^{2} \tan ^{2} \beta,
$$

where $c_{\mu} \simeq 0.5$. Dividing this by the total decay rate of the muon, $\Gamma(\mu) \simeq \Gamma(\mu \rightarrow e \nu \bar{\nu}) \simeq$ $\left(\alpha^{2} / 384 \pi \sin ^{4} \theta_{w} \cos ^{4} \theta_{w}\right)\left(m_{\mu}^{5} / M_{Z}^{4}\right)$, we finally obtain the branching ratio for the $\mu \rightarrow e \gamma$ decay in our theory

$$
\operatorname{Br}(\mu \rightarrow e \gamma) \simeq 3 \times 10^{-11}\left(\frac{200 \mathrm{GeV}}{\tilde{m}}\right)^{4}\left(\frac{\left|W_{\tau \mu}^{e}\right|}{0.04}\right)^{2}\left(\frac{\left|W_{\tau e}^{e}\right|}{0.01}\right)^{2}\left(\frac{\tan \beta}{5.0}\right)^{2} .
$$

Here, we have normalized elements of the new mixing matrix $W^{e}$ by the corresponding values in the CKM matrix. This is well motivated because $W^{e}$ comes from a rotation of the right-handed charged leptons $e$, and the rotation of $e$ is expected to be similar to that of the left-handed quarks $q$, which determines the CKM matrix. 
The prediction given in Eq. (45) is very interesting, since it gives a number close to the present experimental bound $\operatorname{Br}(\mu \rightarrow e \gamma) \lesssim 1.2 \times 10^{-11}$ [36]. While we expect an uncertainty of a factor of a few in the estimate of Eq. (45), for example from uncertainties for the value of $m_{\tilde{\tau}}^{2}$ discussed in sub-section 2.2, we can still say that the present $\mu \rightarrow e \gamma$ decay experiment has already probed our theory up to about $\tilde{m} \simeq 200 \mathrm{GeV}(300 \mathrm{GeV})$ for $\tan \beta=5$ (10). Furthermore, a new experiment is under construction at PSI which aims for a sensitivity of $\operatorname{Br}(\mu \rightarrow e \gamma)$ at a $10^{-14}$ level [37]. Since $\operatorname{Br}(\mu \rightarrow e \gamma) \lesssim 10^{-14}$ corresponds to $\tilde{m} \gtrsim 1.5 \mathrm{TeV}$ $(2 \mathrm{TeV})$ for $\tan \beta=5$ (10) in Eq. (45), it will probe essentially all the parameter region of our theory where radiative electroweak symmetry breaking occurs naturally.

We now discuss the effect of a possible mass splitting between $\tilde{l}_{1,2}$ and $\tilde{l}_{3}$. In deriving a general expression for the lepton flavor violation in Eq. (38), we have used the fact that the masses for $\tilde{l}_{i}$ are all degenerate in our theory, $m_{\tilde{l}_{1,2}}^{2}=m_{\tilde{l}_{3}}^{2}$. However, this relation is not strictly correct; in particular, if $\tan \beta$ becomes larger, $m_{\tilde{l}_{3}}^{2}$ becomes smaller than $m_{\tilde{l}_{1,2}}^{2}$ due to the renormalization group effect through the tau Yukawa coupling. The non-degeneracy between $m_{\tilde{l}_{1,2}}^{2}$ and $m_{\tilde{l}_{3}}^{2}$ introduces new lepton flavor mixing angles in addition to those in $W^{e}$, and causes new diagrams for the $\mu \rightarrow e \gamma$ process. ${ }^{[2}$ Since the amplitudes of these new diagrams can be proportional to $m_{\tau}$ rather than $m_{\mu}$, they might dominate the contribution from the diagrams of Fig. 1 , when $\tan \beta$ is large. The relative size of this new contribution to the previous one is expected to be of order $\left(m_{\tau} / m_{\mu}\right) \delta_{\tilde{l}}$ in the amplitudes, where $\delta_{\tilde{l}} \equiv\left(m_{\tilde{l}_{1,2}}^{2}-m_{\tilde{l}_{3}}^{2}\right) / m_{\tilde{l}_{1,2}}^{2}$. There may also be an additional suppression by a factor of $\epsilon$ if the charged lepton mass matrix is given by the form of Eq. (34), because the relevant rotation angles for $l_{i}$ are then of order $\epsilon^{2}$ while those for $e_{i}$ are of order $\epsilon$. In any event, since $\delta_{\tilde{l}} \simeq 0.02(0.05)$ for $\tan \beta=10(15)$, we find that the contribution from $\delta_{\tilde{l}} \neq 0$ can be safely neglected in the parameter region of our interest, $\tan \beta \lesssim 15$, and the branching ratio of $\mu \rightarrow e \gamma$ is still given by Eq. (45), which is described by the single mixing matrix $W^{e}$. Potentially, the presence of Dirac neutrino Yukawa couplings at high energies could also introduce a splitting between $m_{\tilde{l}_{1,2}}^{2}$ and $m_{\tilde{l}_{3}}^{2}$, enhancing the decay rate of $\mu \rightarrow e \gamma$ [38]. We consider an explicit model of neutrino mass generation in sub-section 5.1, where the neutrino Dirac Yukawa couplings are suppressed by the volume of the extra dimension and their effects on the lepton flavor violating processes are sufficiently small.

The above discussion also tells us something about the location of matter in the extra

11 Even if additional contributions to $m_{\tilde{\tau}}^{2}$, for example from a large $U(1)_{X}$ gauge coupling, happened to give a cancellation between the two diagrams of Fig. đ4b and Fig. 车, reducing $\operatorname{Br}(\mu \rightarrow e \gamma)$ by a few orders of magnitude, the $\mu$-e conversion in nuclei, discussed in the next sub-section, will provide an effective probe of the theory 35 .

12 The situation is somewhat similar to lepton flavor violation in $4 \mathrm{D}$ supersymmetric $S O(10)$ theories, where both $\tilde{l}$ and $\tilde{e}$ non-degeneracies arising from radiative corrections involving the large Yukawa coupling for the third generation 34. 
dimension. Suppose we put the first two generation $\overline{5}$ 's, $F_{1,2}$, in the $5 \mathrm{D}$ bulk rather than on

the $y=0$ brane. In this case, $\tilde{l}_{1,2}$ and $\tilde{l}_{3}$ have a mass splitting of $\delta_{\tilde{l}}=O(1)$ at tree level, so that we cannot go to a basis like Eq. (34) without introducing a flavor violation in the slepton mass-squared matrix. Then, from the structure of the charged lepton Yukawa matrix, we find that the relevant rotation angles for $l_{i}$ are of order $\epsilon$ and the same order with those for $e_{i}$. This means that the $\mu \rightarrow e \gamma$ decay rate in the bulk $F_{1,2}$ theory is enhanced by a factor of $\left(m_{\tau} / m_{\mu}\right)^{2}$ compared with the case of the brane $F_{1,2}$ theory. Therefore, to evade an experimental constraint, the overall mass scale for the superparticles, $\tilde{m}$, in the bulk $F_{1,2}$ theory must be a factor of $\left(m_{\tau} / m_{\mu}\right)^{1 / 2} \simeq 4$ higher than that of the brane $F_{1,2}$ theory. Since the higher value of $\tilde{m}$ requires a fine tuning for electroweak symmetry breaking, the case of $F_{1,2}$ having a different location than $F_{3}\left(m_{\tilde{l}_{1,2}} \neq m_{\tilde{l}_{3}}\right)$ is strongly disfavored, explaining the unique choice for the matter location we made in sub-section 2.2 .

\subsubsection{Other lepton flavor violating processes}

In this sub-section we discuss other lepton flavor violating processes. We first consider the branching ratio for the $\mu \rightarrow 3 e$ decay. This process is dominated by the contribution from photon penguin diagrams, since they are enhanced by the phase space integral. Thus there is a simple relation between $\operatorname{Br}(\mu \rightarrow 3 e)$ and $\operatorname{Br}(\mu \rightarrow e \gamma)$. Using Eq. (45), we obtain

$$
\begin{aligned}
\operatorname{Br}(\mu \rightarrow 3 e) & \simeq \frac{\alpha}{3 \pi}\left(\ln \frac{m_{\mu}^{2}}{m_{e}^{2}}-\frac{11}{4}\right) \operatorname{Br}(\mu \rightarrow e \gamma) \\
& \simeq 2 \times 10^{-13}\left(\frac{200 \mathrm{GeV}}{\tilde{m}}\right)^{4}\left(\frac{\left|W_{\tau \mu}^{e}\right|}{0.04}\right)^{2}\left(\frac{\left|W_{\tau e}^{e}\right|}{0.01}\right)^{2}\left(\frac{\tan \beta}{5.0}\right)^{2} .
\end{aligned}
$$

The present experimental bound is $\operatorname{Br}(\mu \rightarrow 3 e) \lesssim 1.0 \times 10^{-12}$ [39].

We next consider $\mu \rightarrow e$ conversion in nuclei. The conversion rate is well approximated by

$$
\Gamma(\mu \rightarrow e ; X) \simeq 16 \alpha^{4} Z_{\mathrm{eff}}^{4} Z|F(q)|^{2} \Gamma(\mu \rightarrow e \gamma)
$$

in the parameter region we are considering. (The approximation is better for larger values of $\tan \beta$.) Here, $Z$ represents the proton number of the nucleus $X ; Z_{\text {eff }}$ and $F(q)$ are the effective charge and the nuclear form factor at the momentum transfer $q$, respectively. In the case of $X={ }_{22}^{48} \mathrm{Ti}$, for which $Z=22, Z_{\text {eff }} \simeq 17.6$ and $|F(q)| \simeq 0.54$ [40, we obtain the prediction for the $\mu \rightarrow e$ conversion rate, normalized by the muon capture rate $\Gamma\left(\mu \rightarrow\right.$ capture; $\left.{ }_{22}^{48} \mathrm{Ti}\right)=$ $(2.590 \pm 0.012) \times 10^{6} \mathrm{sec}^{-1}$ 41, of

$$
\begin{aligned}
\operatorname{Cr}\left(\mu \rightarrow e ;_{22}^{48} \mathrm{Ti}\right) & \equiv \frac{\Gamma\left(\mu \rightarrow e ;_{22}^{48} \mathrm{Ti}\right)}{\Gamma\left(\mu \rightarrow \text { capture }{ }_{22}^{48} \mathrm{Ti}\right)} \\
& \simeq 2 \times 10^{-13}\left(\frac{200 \mathrm{GeV}}{\tilde{m}}\right)^{4}\left(\frac{\left|W_{\tau \mu}^{e}\right|}{0.04}\right)^{2}\left(\frac{\left|W_{\tau e}^{e}\right|}{0.01}\right)^{2}\left(\frac{\tan \beta}{5.0}\right)^{2}
\end{aligned}
$$


The present experimental bound is $\operatorname{Cr}\left(\mu \rightarrow e ;{ }_{22}^{48} \mathrm{Ti}\right) \lesssim 4.3 \times 10^{-12}$ [42]. It is interesting to note that future experiments may probe $\mu \rightarrow e$ conversion in nuclei with a sensitivity below $10^{-16}$ 43.

Another important lepton flavor violating process is $\tau \rightarrow \mu \gamma$ decay. The branching ratio for $\tau \rightarrow \mu \gamma$ is related to that of $\mu \rightarrow e \gamma$ by

$$
\frac{\Gamma(\tau \rightarrow \mu \gamma)}{\Gamma(\mu \rightarrow e \gamma)}=\left|\frac{W_{\tau \tau}^{e}}{W_{\tau e}^{e}}\right|^{2} \operatorname{Br}(\tau \rightarrow \mu \nu \bar{\nu})
$$

Since $\operatorname{Br}(\tau \rightarrow \mu \nu \bar{\nu})=(17.37 \pm 0.07) \%$, we obtain

$$
\operatorname{Br}(\tau \rightarrow \mu \gamma) \simeq 5 \times 10^{-8}\left(\frac{200 \mathrm{GeV}}{\tilde{m}}\right)^{4}\left(\frac{\left|W_{\tau \mu}^{e}\right|}{0.04}\right)^{2}\left(\frac{\left|W_{\tau \tau}^{e}\right|}{1.0}\right)^{2}\left(\frac{\tan \beta}{5.0}\right)^{2}
$$

The present experimental bound comes from CLEO: $\operatorname{Br}(\tau \rightarrow \mu \gamma) \lesssim 1.1 \times 10^{-6}$ [44]. The $B$ factories at KEK and SLAC will improve the bound to the level of $10^{-7}$. Note that the combination of lepton flavor violation mixing angles, $\theta_{i j}^{e}$, appearing in Eq. (50) is different from that in Eqs. (45, 46, 48). Therefore, in principle, we can determine all the lepton flavor violation mixing angles, $\theta_{12}^{e}$ and $\theta_{23}^{e}$, by measuring both $\mu \rightarrow e$ and $\tau \rightarrow \mu$ transition rates, if we know $\tilde{m}$ and $\tan \beta$ from independent measurements of the superparticle spectrum.

\subsection{Hadronic flavor violation}

The structure of hadronic flavor violation in our theory is much more complicated than that of lepton flavor violation. In addition to the complication arising from the CKM matrix, there is also a complication coming from the fact that up-type squarks have flavor non-universalities in both the left-handed and right-handed mass-squared matrices. Although it is still possible to derive a general Lagrangian for hadronic flavor violation, as in the leptonic case of Eq. (38), the resulting expression is not particularly illuminating, so that here we focus on estimating constraints on the overall superparticle mass scale, $\tilde{m}$, coming from various hadronic flavor violating processes.

We start with the $b \rightarrow s \gamma$ decay process. In the minimal supergravity scenario of supersymmetry breaking, it is often claimed that the exact $b / \tau$ unification is consistent with the constraint from the $b \rightarrow s \gamma$ decay only when the squark masses are rather large. This is because the exact $b / \tau$ unification requires the negative sign of $\mu$ and/or large values for $\tan \beta$, which enhances the supersymmetric contribution to the $b \rightarrow s \gamma$ process. In our theory, however, there is a correction from the unified scale, Eq. (28), allowing us to consider the parameter region of $\tan \beta \simeq 5-10$. Thus the squark masses do not have to be very large. Furthermore, the $b \rightarrow s \gamma$ decay is a rigid constraint on the parameter space only if squark mass matrices are diagonal 
in the super-CKM basis. This is not the case in our theory, since the squark mass matrices have flavor non-universalities at the compactification scale. By rotating to the super-CKM basis, this introduces flavor off-diagonal elements in the squark mass matrices, which are not determined by the observed CKM mixing angles. Therefore, in our theory we cannot extract a definite bound from the $b \rightarrow s \gamma$ process. A rough bound is obtained as $\tilde{m} \gtrsim 100 \sqrt{\tan \beta} \mathrm{GeV}$ by estimating the size of the chargino exchange diagrams.

We next consider constraints from neutral meson mixings. Since flavor violation in the squark sector is relatively small due to the gluino focusing effect, we can use the mass insertion approximation to estimate flavor violating processes. The relevant quantities are the mass insertion parameters $\delta_{i j}$ defined by $\left(\delta_{i j}^{f}\right)_{X Y} \equiv\left(m_{\tilde{f}}^{2}\right)_{i X, j Y} / \sqrt{\left(m_{\tilde{f}}^{2}\right)_{i X, i X}\left(m_{\tilde{f}}^{2}\right)_{j Y, j Y}}$ in the super-CKM basis, where $f=u, d$ specifies the up-type or down-type sector and $X, Y=$ $L, R$ the left-handed or right-handed chirality; $i, j=1,2,3$ are the generation indices. The $K_{L}-K_{R}$ mass difference, $\Delta m_{K}$, gives constraints on $\left(\delta_{12}^{d}\right)_{X Y}$. In our theory, these quantities are estimated as $\left(\delta_{12}^{d}\right)_{L L} \approx\left(m_{\tilde{q}_{B}}^{2}-m_{\tilde{q}_{3}}^{2}\right) V_{t d}^{\mathrm{CKM}} V_{t s}^{\mathrm{CKM}} / m_{\tilde{q}_{B}}^{2} \approx 10^{-4},\left(\delta_{12}^{d}\right)_{R R} \approx 0$, and $\left(\delta_{12}^{d}\right)_{L R},\left(\delta_{12}^{d}\right)_{R L} \lesssim m_{s} \mu \tan \beta / m_{\tilde{q}_{B}}^{2} \approx 10^{-4} \tan \beta(200 \mathrm{GeV} / \tilde{m})$, assuming no contribution from brane-localized kinetic terms. Therefore, we find that the supersymmetric contribution to $\Delta m_{K}$ is negligible for $\tilde{m} \gtrsim 200 \mathrm{GeV}$ [22]. Since the constraint from the $C P$ violating parameter $\varepsilon_{K}$ is somewhat stronger than that from $\Delta m_{K}$, however, there may be an observable effect on $\varepsilon_{K}$ in the case of lower values for $\tilde{m}$ (and larger $\tan \beta$ ) if the relevant phase is of order unity. Analogous considerations can also be made for $B-\bar{B}$ and $D-\bar{D}$ mixings, which constrain $\left(\delta_{13}^{d}\right)_{X Y}$ and $\left(\delta_{12}^{u}\right)_{X Y}$, respectively. Again, we obtain essentially no constraint for $\tilde{m} \gtrsim 200 \mathrm{GeV}$.

\section{A Realistic Completion of the Theory}

In this section we provide a realistic extension of our theory, incorporating small neutrino masses and a natural generation of the supersymmetric mass term ( $\mu$ term) and the holomorphic supersymmetry breaking mass term ( $\mu B$ term) for the Higgs doublets. In our example, these two issues are related through a single dynamics triggering the spontaneous breaking of the $U(1)_{X}$ gauge symmetry. Small neutrino masses are generated by integrating out right-handed neutrino superfields through the see-saw mechanism [12]. The $\mu$ and $\mu B$ terms of the correct size are generated by the vacuum readjustment mechanism of Ref. [13]. The generated $B$ parameter is real, so that there is no supersymmetric $C P$ problem.

\subsection{Neutrino masses and $U(1)_{X}$ gauge interaction}

Recent neutrino experiments have provided strong evidence that neutrinos have small masses and the different flavors are mixed [45, 46]. In our theory small neutrino masses can be gener- 
ated through the see-saw mechanism by introducing three generations of right-handed neutrino superfields. They could be either brane fields, $N$, or bulk fields, $\left\{N, N^{c}\right\}$ with $\eta_{N}=1$. In both cases, the Yukawa couplings and Majorana masses for $N$ are located on the $y=0$ brane

$$
S=\int d^{4} x d y \delta(y)\left[\int d^{2} \theta\left(y_{N} F N H+\frac{\kappa_{R}}{2} N N\right)+\text { h.c. }\right] .
$$

The $U(1)_{R}$ charges for the right-handed neutrinos are given by $R(N)=R\left(N^{c}\right)=1$, as for the other matter fields $T$ and $F$, so that the above superpotential terms are invariant under $U(1)_{R}$. After integrating over the extra dimension, these terms give the $4 \mathrm{D}$ neutrino Dirac Yukawa couplings, $\left[y_{\nu} L N H_{u}\right]_{\theta^{2}}$, and the $4 \mathrm{D}$ Majorana mass terms $\left[\left(M_{R} / 2\right) N N\right]_{\theta^{2}}$. Thus, integrating out the right-handed neutrinos we obtain the operators $\left[\left(y_{\nu}^{2} / 2 M_{R}\right) L L H H\right]_{\theta^{2}}$ at low energies, which provide small masses $m_{\nu} \sim y_{\nu}^{2} v^{2} / M_{R}$ to the observed (left-handed) neutrinos.

What is the scale for $M_{R}$ ? It depends on the size of the 4D neutrino Dirac Yukawa couplings, $y_{\nu}$. If $N$ is the brane field, we expect that $y_{\nu}=O(1)$ at least for the third generation, so that $M_{R}$ could be as high as the compactification scale, $M_{c}^{\prime}$, keeping $m_{\nu} \simeq 0.03-0.1 \mathrm{eV}$ suggested from the atmospheric neutrino oscillation data. Thus, in this case, the interactions in Eq. (51) will not affect the superparticle mass spectrum much, since the superparticle masses are effectively generated at $M_{c}^{\prime}$. On the other hand, if $N$ is in the bulk, the $4 \mathrm{D}$ Yukawa coupling $y_{\nu}$ is volume suppressed, so that $M_{R}$ will be smaller than $M_{c}^{\prime}$ to obtain desired values for the neutrino masses. Therefore, the neutrino interactions in Eq. (51) could potentially affect the superparticle mass spectrum through renormalization group evolutions. Below, we will choose $N$ to be in the bulk and give an example of the complete theory, but the same mechanism works also for the brane $N$ case with obvious modifications of the statements that are specific to the bulk $N$ case.

We introduce a $U(1)_{X}$ gauge interaction $(\subset S O(10) / S U(5))$ in the bulk, under which matter and Higgs fields transform as $T(1), F(-3), N(5), H(-2)$ and $\bar{H}(2)$ (for the bulk fields, the conjugated chiral superfields have opposite quantum numbers to the corresponding unconjugated fields). Then, the Majorana masses for $N$ are generated by the spontaneous breaking of the $U(1)_{X}$ symmetry, explaining why they take values smaller than the value expected from pure dimensional analysis. One way of realizing this is to introduce $U(1)_{X}$ breaking fields $\Psi(10)$ and $\bar{\Psi}(-10)$ with the following superpotential

$$
S=\int d^{4} x d y \delta(y)\left[\int d^{2} \theta\left(f X\left(a \Psi \bar{\Psi}-\Lambda^{2}\right)+\frac{\kappa}{2} \bar{\Psi} N N\right)+\text { h.c. }\right],
$$

where $X$ is a gauge singlet field, and $\Lambda$ is a scale arising from the dynamics of some strongly coupled gauge interaction.[1] This superpotential forces vacuum expectation values for the $\Psi$

\footnotetext{
${ }^{13}$ An explicit example for the strongly interacting sector is given in Ref. [13], where the $S U(2)_{S}$ gauge interaction with four doublet fields $\mathcal{Q}_{i}$ and five singlet fields $X^{a}$ having an appropriate superpotential is considered. We here assume that this strong sector is localized on the $y=0$ brane, which explains why this gauge interaction is stronger than the other gauge interactions such as the standard model ones and $U(1)_{X}$.
} 
and $\bar{\Psi}$ fields, $\langle\Psi\rangle=\langle\bar{\Psi}\rangle=\Lambda / \sqrt{a}$, giving Majorana masses for the right-handed neutrinos, $\kappa_{R}=\kappa \Lambda / \sqrt{a}$. Thus the superpotential Eq. (52) effectively reproduces the second term of the superpotential Eq. (51). Note that the whole sector of neutrino mass generation is invariant under $U(1)_{R}$. Specifically, the $U(1)_{R}$ charge assignment for various fields is given by $R(X)=2$ and $R(\Psi)=R(\bar{\Psi})=0$, and $U(1)_{R}$ is not broken by the dynamics of this sector. TH

We finally comment on the possible effect of the interactions in Eq. (51) on the superparticle spectrum. In the case that $N$ propagates in the bulk, the $4 \mathrm{D}$ neutrino Yukawa coupling $y_{\nu}$ receives a volume suppression and is expected to be $y_{\nu} \lesssim \epsilon \approx 0.1$. Therefore, the effect of this coupling on the low energy spectrum will be small. In particular, the splitting between $m_{\tilde{l}_{1,2}}$ and $m_{\tilde{l}_{3}}$ caused by this coupling through the renormalization group evolution is expected to be sufficiently small that the previous estimates for lepton flavor violating processes will remain intact. On the other hand, smaller Yukawa couplings suggest a smaller scale for the Majorana mass $M_{R}$. However, this does not necessarily mean that the scale for the $U(1)_{X}$ breaking is small. In fact, by tracing the volume suppression factors, we find that the vacuum expectation values for $\Psi$ and $\bar{\Psi}$ are not much smaller than the compactification scale. Therefore, the radiative contribution to the stau mass from the $U(1)_{X}$ gaugino is also expected to be small.'.0

\section{$5.2 \quad$ Origin of $\mu$ term}

The neutrino mass generation of the previous sub-section also provides a natural mechanism for generating the $\mu$ term [13]. One easy way to implement this mechanism is to put the $\Psi$ and $\bar{\Psi}$ fields in the $5 \mathrm{D}$ bulk as two hypermultiplets, $\left\{\Psi, \Psi^{c}\right\}+\left\{\bar{\Psi}, \bar{\Psi}^{c}\right\}$ with $\eta_{\Psi}=\eta_{\bar{\Psi}}=1$, having the superpotential coupling Eq. (52) on the $y=0$ brane. Now, suppose that the scale of the vacuum expectation values for $\Psi$ and $\bar{\Psi}$ is lower than the compactification scale. This occurs in some parameter region of the theory, for example, if the dimensionless coefficients of the neutrino Yukawa couplings are somewhat smaller than the other couplings. In this case, the $4 \mathrm{D}$ effective theory below $M_{c}^{\prime}$ contains holomorphic supersymmetry breaking terms in addition to the supersymmetric terms arising from Eq. (52). Using the general result of Eq. (16), we find that holomorphic supersymmetry breaking parameters for $X \Psi \bar{\Psi}$ and $X \Lambda^{2}$ terms are different $(-2 \tilde{m}$ and 0 in the case that $X$ is a brane field). Then, by minimizing the scalar potential, we find that the $X$ superfield develops vacuum expectation values of order the weak scale in both lowest and highest components, $\langle X\rangle \sim \tilde{m}$ and $\left\langle F_{X}\right\rangle \sim \tilde{m}^{2}$. Therefore, by introducing the

\footnotetext{
${ }^{14}$ In the $S U(2)_{S}$ example of Ref. [13], $R\left(X^{a}\right)=2$ and $R\left(\mathcal{Q}_{i}\right)=0$ so that $U(1)_{R}$ does not have an anomaly for $S U(2)_{S}$ (i.e. $\left.R(\Lambda)=0\right)$.

15 Such expectations are not definitive: there is a possibility that this correction is sizable due to longer running distances and/or larger values of the $U(1)_{X}$ gauge coupling.
} 
coupling

$$
S=\int d^{4} x d y \delta(y)\left[\int d^{2} \theta \lambda X H \bar{H}+\text { h.c. }\right],
$$

we obtain the $\mu$ and $\mu B$ terms of the correct size. Since the $B$ parameter generated through this mechanism is real, there is no supersymmetric $C P$ problem. This means that the $\mu$ parameter is real in our phase convention where $\tan \beta$ (and thus the $\mu B$ parameter) is taken to be real. Note that the above coupling in Eq. (53) respects the $U(1)_{R}$ symmetry, so that the whole system is still $U(1)_{R}$ invariant. The $U(1)_{R}$ breaking lies only in the supersymmetry breaking terms arising from the boundary conditions, which can be viewed as a vacuum expectation value for an auxiliary field in the 5D gravity multiplet.

Finally, we briefly comment on an alternative possibility of generating the $\mu$ term. Instead of relying on the above vacuum readjustment mechanism, we could introduce the singlet field $S$ at the weak scale and write down the superpotential terms $\left[S H \bar{H}+S^{3}\right]_{\theta^{2}}$ on the $y=0$ brane. Then, in some parameter region, the lowest and highest components of the $S$ field get vacuum expectation values of the order of the weak scale, generating $\mu$ and $\mu B$ terms of the correct order 477. In this case the $U(1)_{R}$ symmetry is explicitly broken to the discrete $Z_{4, R}$ subgroup by the $S^{3}$ term in the superpotential, but it is still sufficient to suppress unwanted terms such as tree-level $\mu$ term and dimension four and five proton decay operators. Since the Higgs quartic couplings receive an additional contribution from the superpotential term $S^{3}$, the physical Higgs boson mass can be larger than that in the MSSM-type models where there is no singlet field around the weak scale.

\section{Alternative Possibility}

We have seen that the location of matter in our theory is uniquely determined as a consequence of the "large" extra dimension and by the requirements of $b / \tau$ unification and naturalness for electroweak symmetry breaking. The predictive framework for gauge coupling unification requires strong coupling at the cutoff scale, and thus the large volume for the extra dimension. The location for $T_{1}$ and $T_{3}$ are then determined to be the bulk and the brane by considering the constraint from dimension six proton decay and the size of the top Yukawa coupling, respectively. Breaking supersymmetry by boundary conditions, the first two generations having the same gauge quantum numbers must be located in the same place to evade constraints from flavor changing neutral current processes; hence $T_{2}$ must be located in the bulk. The $b / \tau$ unification requires $F_{3}$ on the brane, and finally $F_{1,2}$ are located in the same place as $F_{3}$ to avoid too large lepton flavor violating processes.

Obviously, relaxing some of these requirements allow us to consider other possibilities for the matter location, which we explore in this section in the framework of KK grand unification 
with boundary condition supersymmetry breaking. We first observe that if we insist on the predictive scheme for gauge coupling unification, namely the strong coupling scenario, the location of $T_{i}$ are completely fixed: $T_{3}$ on the brane and $T_{1,2}$ in the bulk. Then we find that all $F_{i}$ must be put together in the same place to evade excessive lepton flavor violating processes which would push up the overall mass scale for superpartners. Therefore, we have only two choices for the location of $F_{i}$ : all $F_{i}$ on the brane, which we have adopted so far in this paper, or all $F_{i}$ in the bulk. In the latter case of $F_{i}$ in the bulk, there is no $b / \tau$ unification because the bottom and tau Yukawa couplings come from different interactions that are not related by the $S U(5)$ symmetry. However, we instead obtain an understanding of the $t / b$ mass ratio, since $T F \bar{H}$ type Yukawa couplings are now suppressed by the volume factor compared with TTH type couplings. Thus we find that the case of bulk $F_{i}$ is also interesting, especially if future improvements of extracting $m_{b}$ from data determine $m_{b}$ to be in the lower part of the presently allowed experimental region.

The superparticle spectrum in the case of bulk $F_{i}$ is different from that of the brane $F_{i}$ case, corresponding to the difference of soft supersymmetry breaking parameters at the compactification scale. In particular, we now have to use the values of $\tilde{d}_{B}$ and $\tilde{l}_{B}$ in Table 3 for the first two generation $\tilde{d}_{1,2}$ and $\tilde{l}_{1,2}$, instead of $\tilde{d}_{b}$ and $\tilde{l}_{b}$. Similarly, the values for the third generation squark and slepton masses are changed from $\left(\tilde{q}_{3}, \tilde{u}_{3}, \tilde{d}_{3}, \tilde{l}_{3}, \tilde{e}_{3}\right) \simeq(390,310,420,140,66)$ to $(390,310,460,240,63)$ in Table 3 . The sizes for the $A$ terms are also changed: $\left(A_{t}, A_{b}, A_{\tau}\right) \simeq$ $(-410,-730,-320) \rightarrow(-410,-930,-520)$. These changes affect the expression of the weak scale threshold correction to gauge coupling unification. The new expression is given by $\left.\delta \alpha_{s}\right|_{\text {susy }} ^{\mathrm{KK}} \simeq 0.0034-0.0030 \ln \left(\tilde{m} / M_{Z}\right)$ instead of Eq. (23), which makes the prediction for the QCD coupling slightly lower than the brane $F_{i}$ case for the same value of $\tilde{m}$. Note also that the bulk $F_{i}$ theory allows both signs for the $\mu$ parameter, since the supersymmetric threshold correction to $m_{b}$ can now have either sign.

Proton decay in the bulk $F_{i}$ theory is very much suppressed. The decay by flavor mixings receives the suppression of order $\left(V_{13} V_{32}\right)^{2}$ in the amplitude, giving the lifetime estimate $\tau(p \rightarrow$ $\left.\mu^{+} K^{0}\right) \approx 10^{44}$ years. The decay through brane kinetic operators is also suppressed: $\left(M_{c}^{\prime} / M_{s}\right)^{2}$ suppression in the amplitude, giving $\tau\left(p \rightarrow e^{+} \pi^{0}, \cdots\right) \approx 10^{39}$ years.

The rates for lepton flavor violating processes are also subject to important changes. We first consider the contributions to the $\mu \rightarrow e \gamma$ process proportional to tan $\beta$, which come from the diagrams of Fig. 凹b and Fig. ⿶cc. In the theory where $F_{i}$ are on the brane, the diagram

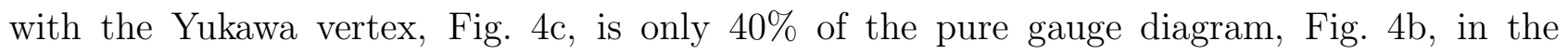
amplitude. However, in the theory with bulk $F_{i}$, the left-handed sleptons are heavier, making the contribution from the diagram of Fig. đb smaller. By explicitly calculating the two diagrams, we find that the two contributions have a comparable size with the opposite sign. Hence the 
potentially leading contribution with the $\tan \beta$ enhancement turns out to be small due to the cancellation between the two diagrams. The precise calculation for the value of the remaining contribution is difficult without a precise knowledge of the superparticle mass spectrum, but we can roughly estimate the expected size for $\operatorname{Br}(\mu \rightarrow e \gamma)$ by evaluating the piece which is not proportional to $\tan \beta$. This piece is larger than the previous case because the $A$ terms are larger due to the bulk location of $F_{i}$; specifically, the coefficient in Eq. (43) becomes $0.2 \rightarrow 1$. Overall, we expect lepton flavor violation rates in the bulk $F_{i}$ theory to be one or two orders of magnitude smaller than the corresponding ones in the brane $F_{i}$ theory. However, since the parameter region which leads to the exact cancellation is different for different processes, for instance between the $\mu \rightarrow e \gamma$ decay and the $\mu$-e conversion in nuclei, some of them will survive the strong cancellation and could still have comparable rates to the case of the brane $F_{i}$ theory.

We finally comment on the possibility of relaxing the strong coupling assumption at $M_{s}$. In this case the volume of the extra dimension does not necessarily have to be very large, so we can consider yet other possibilities for the matter location, although we loose the high predictivity for gauge coupling unification leading to the prediction as Eq. (24). In particular, the location of $T_{i}$ is now not completely determined. The cases where all matter fields are located on the brane or in the bulk have been discussed in Ref. [10].

\section{Conclusions}

The merging of gauge couplings at energies of order $10^{16} \mathrm{GeV}$ heralds some new unified physics beyond the supersymmetric desert. An attractive new option for this physics is that extra dimensions of spacetime are resolved, with local defects explicitly breaking the unified gauge symmetry [1, 2]. Advances of 4D grand unification are kept, while many of the problems are overcome. An understanding of quark and lepton quantum numbers is preserved, while gauge coupling unification emerges in the limit that the defects are embedded in a large bulk. The breaking of gauge symmetry is automatic, as is a large mass gap between light and heavy gauge fields and between doublet and triplet Higgs fields [3]. Proton stability from operators of dimension four and five is guaranteed by a continuous $R$ symmetry of the underlying 5D supersymmetric theory, while quark-lepton mass relations are only expected for heavy generations. A crucial question for this new framework is: how can it be tested?

In this paper we have developed the minimal KK grand unified theory of Ref. [2], based on $S U(5)$ gauge interactions in 5D, into a complete, realistic theory. The major new ingredient is to break supersymmetry by boundary conditions applied to the same fifth dimension that breaks the gauge symmetry. If this is the correct effective theory of nature, over the next decade experiments will provide convincing evidence for it, measuring the locations of quarks and 
leptons in the bulk. While it seems to us a natural way to incorporate supersymmetry breaking into KK unified theories, there are clearly other possibilities, which will lead to alternative phenomenologies. Having made this choice for supersymmetry breaking, the location of each quark and lepton field in the fifth dimension is unique, up to a two-fold ambiguity, definite predictions for both the superpartner spectrum and for lepton flavor violation.

The heart of our predictions rests on their being a single supersymmetry breaking boundary condition parameter, $\tilde{m}$, so that, even allowing for arbitrary $\mu$ and $B$ parameters in the Higgs potential, the entire superpartner spectrum depends on only $\tilde{m}$ and $\tan \beta$. Examples of this spectrum for two values of $(\tilde{m}, \tan \beta)$ are shown in Table 4 . The range of $\tilde{m}$ and $\tan \beta$ is limited: $\tilde{m}$ cannot be much less than $200 \mathrm{GeV}$ from the experimental limit on the mass of the charged scalar tau, and should not be much more than about $500 \mathrm{GeV}$, since above this electroweak symmetry breaking rapidly becomes more fine tuned. The ratio of electroweak vacuum expectation values, $\tan \beta$, has a lower bound of about 3 from the limit on the Higgs boson mass, and must be less than about 25 to ensure that the mass squared for the righthanded scalar tau is positive. It is clear that the precision of the predictions for the entire spectrum in terms of these two parameters allows a very significant test of many aspects of the theory. In particular, the superpartners which reside on the brane, those in $T_{3}$ and $F_{1,2,3}$, have zero tree-level mass and get heavy only from renormalization group scaling. This is particularly clear in the sleptons, and is visible in the lightness of $\tilde{\tau}_{1,2}$ and $\tilde{l}_{1,2}$. These effects are also present in the squarks, although this is somewhat hidden by the gluino focusing effect. The mass eigenstates of the third generation squarks are not given by the soft mass-squared parameters for the helicity eigenstates because of the mixing induced by important $A$ parameters, which take the value $A_{t}=A_{b}=A_{\tau}=-\tilde{m}$ at the compactification scale, reflecting the Higgs residing in the bulk and the third generation on the brane. A detailed study of the superpartner and Higgs spectrum would thus not only measure $\tilde{m}$ and $\tan \beta$, but would also verify the location of each matter and Higgs field.

The predictions of our theory for $\alpha_{s}\left(M_{Z}\right)$ from gauge coupling unification and the $b$ quark mass from Yukawa unification are

$$
\begin{aligned}
& \alpha_{s}\left(M_{Z}\right)=\left(0.1327-0.0030 \ln \frac{\tilde{m}}{M_{Z}}-0.0019 \ln \frac{M_{s}}{M_{c}^{\prime}}\right) \pm 0.003 \\
& m_{b}\left(M_{Z}\right)=\left(3.62-0.022 \tan \beta-0.026 \ln \frac{M_{s}}{M_{c}^{\prime}}\right) \pm 0.1 \mathrm{GeV} .
\end{aligned}
$$

In the above expression, the leading term is given first, the second term is the supersymmetric threshold correction and the third term is the unified scale correction; the uncertainty for $\alpha_{s}$

\footnotetext{
${ }^{16}$ The two cases correspond to whether the five-plets, $F_{i}$, are in the bulk or on a brane. We prefer the brane case, since only then does a unified prediction for $m_{b} / m_{\tau}$ follow, and quote predictions for this case in the conclusions.
} 


\begin{tabular}{|c|c|c|}
\hline$\tilde{m}$ & 300 & 400 \\
$\tan \beta$ & 5 & 10 \\
\hline$\tilde{g}$ & 699 & 911 \\
$\tilde{\chi}_{1}^{ \pm}$ & 251 & 334 \\
$\tilde{\chi}_{2}^{ \pm}$ & 427 & 531 \\
$\tilde{\chi}_{1}^{0}$ & 130 & 175 \\
$\tilde{\chi}_{2}^{0}$ & 251 & 334 \\
$\tilde{\chi}_{3}^{0}$ & 417 & 518 \\
$\tilde{\chi}_{4}^{0}$ & 422 & 528 \\
\hline$\tilde{q}$ & 701 & 915 \\
$\tilde{u}$ & 675 & 880 \\
$\tilde{d}$ & 602 & 780 \\
$\tilde{l}$ & 209 & 277 \\
$\tilde{e}$ & 317 & 422 \\
\hline$\tilde{t}_{1}$ & 425 & 547 \\
$\tilde{t}_{2}$ & 619 & 780 \\
$\tilde{b}_{1}$ & 563 & 727 \\
$\tilde{b}_{2}$ & 601 & 774 \\
$\tilde{\tau}_{1}$ & 106 & 126 \\
$\tilde{\tau}_{2}$ & 214 & 280 \\
\hline$h$ & 118 & 128 \\
$A$ & 552 & 690 \\
$H^{0}$ & 553 & 690 \\
$H^{ \pm}$ & 558 & 695 \\
\hline $\operatorname{Cr}\left(\mu \rightarrow e ;{ }_{22} \operatorname{Ti}\right)$ & $4 \times 10^{-14}$ & $5 \times 10^{-14}$ \\
$\operatorname{Br}(\tau \rightarrow \mu \gamma)$ & $1 \times 10^{-8}$ & $1 \times 10^{-8}$ \\
\hline
\end{tabular}

Table 4: Predictions for the superpartner spectrum, the Higgs spectrum, gauge and Yukawa unification, and lepton flavor violating processes. The predictions are for two representative values of $\tilde{m}$ and $\tan \beta$, and all masses are given in $\mathrm{GeV}$. Mass eigenvalues are given for the gluino, $\tilde{g}$, the charginos, $\tilde{\chi}^{ \pm}$, the neutralinos, $\tilde{\chi}^{0}$, the squarks and sleptons of the third generation, $\tilde{t}_{1,2}, \tilde{b}_{1,2}$ and $\tilde{\tau}_{1,2}$, and the Higgs bosons, $h, A, H^{0}$ and $H^{ \pm}$. The mass of the lightest Higgs boson, $h$, includes one-loop radiative corrections from top quarks and squarks. For the first two generations of squarks and sleptons the masses are shown for $\tilde{q}, \tilde{u}, \tilde{d}, \tilde{l}$ and $\tilde{e}$ and do not include contributions from electroweak $D$ terms. 
arises from unknown physics at and above $M_{s}$. These are remarkably precise predictions. The supersymmetric threshold corrections involve the two parameters $\tilde{m}$ and $\tan \beta$, have relatively small coefficients, and will be known once superpartner masses are measured. The assumption of strong 5D gauge interactions at $M_{s}$ leads to the prediction $M_{s} / M_{c}^{\prime} \approx 200$. It is quite remarkable that the leading unified scale corrections are thus calculable and predicted, and move both predictions into agreement with experimental data. Using $\tilde{m}=400 \mathrm{GeV}$ and $\tan \beta=10$, we obtain $\alpha_{s}\left(M_{Z}\right)=0.118 \pm 0.003$ and $m_{b}\left(M_{Z}\right)=3.3 \pm 0.1 \mathrm{GeV}$. The prediction for the QCD coupling is in precise agreement with data, unlike the case of conventional supersymmetric grand unification, where large uncalculable unified threshold corrections are required. We predict $m_{b}\left(M_{Z}\right)$ to be at the upper end of the presently allowed experimental region of $3.0 \pm 0.3 \mathrm{GeV}$.

Lepton flavor violation is an important experimental signal for conventional supersymmetric unified theories 33, 34, providing soft supersymmetry breaking operators are local at the unified scale. The slepton non-universality arises from renormalization group scaling from the top quark Yukawa coupling above the unification scale. Uncertainties arise from two sources, flavor mixing matrices and the structure of the theory above the unified scale. Slepton nondegeneracy could also arise from scaling from the neutrino Yukawa coupling below the unified scale [38]. In this paper we have demonstrated that lepton flavor violation is also an important signature in our theory. However, in contrast to 4D theories, slepton non-degeneracy arises at tree level and is maximal. A heavy top quark results from $T_{3}$ being located on the brane, so that, at tree level, $\tilde{\tau}_{R}$ does not feel the supersymmetry breaking boundary condition and is massless. By contrast $T_{1}$ must be located in the bulk, otherwise gauge boson exchange leads to too large a proton decay rate, so that $m_{\tilde{e}_{R}}=\tilde{m}$ at the compactification scale. This maximal slepton nondegeneracy leads to larger rates for lepton flavor violation in our $5 \mathrm{D}$ theory than in conventional supersymmetric unified theories, and furthermore reduces the uncertainties of the signal. While there is still a dependence on the flavor mixing matrices, the uncertainties associated with the generation of the slepton non-degeneracy is removed. The first immediate consequence is that $T_{2}$ must be located with $T_{1}$ in the bulk, to avoid a large non-degeneracy between $\tilde{e}$ and $\tilde{\mu}$, and the three $F_{i}$ must have a common location to avoid too large lepton flavor violation from diagrams involving non-degeneracies in both left and right-handed sleptons. With $F_{i}$ on the brane, the branching ratios for flavor violating lepton decays are found to be close to the present experimental limits

$$
\begin{aligned}
& \operatorname{Br}(\mu \rightarrow e \gamma) \simeq 3 \times 10^{-11}\left(\frac{200 \mathrm{GeV}}{\tilde{m}}\right)^{4}\left(\frac{\left|W_{\tau \mu}^{e}\right|}{0.04}\right)^{2}\left(\frac{\left|W_{\tau e}^{e}\right|}{0.01}\right)^{2}\left(\frac{\tan \beta}{5.0}\right)^{2}, \\
& \operatorname{Br}(\mu \rightarrow 3 e) \simeq \operatorname{Cr}\left(\mu \rightarrow e ;{ }_{22}^{48} \mathrm{Ti}\right) \simeq 2 \times 10^{-13}\left(\frac{200 \mathrm{GeV}}{\tilde{m}}\right)^{4}\left(\frac{\left|W_{\tau \mu}^{e}\right|}{0.04}\right)^{2}\left(\frac{\left|W_{\tau e}^{e}\right|}{0.01}\right)^{2}\left(\frac{\tan \beta}{5.0}\right)^{2}(5,7)
\end{aligned}
$$




$$
\operatorname{Br}(\tau \rightarrow \mu \gamma) \simeq 5 \times 10^{-8}\left(\frac{200 \mathrm{GeV}}{\tilde{m}}\right)^{4}\left(\frac{\left|W_{\tau \mu}^{e}\right|}{0.04}\right)^{2}\left(\frac{\left|W_{\tau \tau}^{e}\right|}{1.0}\right)^{2}\left(\frac{\tan \beta}{5.0}\right)^{2}
$$

Once $\tilde{m}$ and $\tan \beta$ are determined from the superpartner spectrum, observation of these decay modes would measure the two independent flavor mixing angles of the lepton mixing matrix $W^{e}$. Indirect evidence for our theory would follow if the size of this intergenerational mixing is comparable to that measured in the quark sector. To go further would require a more detailed theory of flavor than we have given here.

We stress that large lepton flavor violation is a generic signature in any KK grand unified theory where the supersymmetry breaking reflects the structure of matter location in extra dimensions. The matter locality breaks $U(3)$ flavor symmetry, leading to squark and slepton mass matrices of the general form of Eq. (32) dictated by the flavor symmetry of the 5D gauge interactions. The low energy superparticle spectrum then reveals characteristic features reflecting the structure of this new flavor symmetry, irrespective of how supersymmetry is broken. Therefore, lepton flavor violation probes all supersymmetry breaking schemes which gives soft operators local up to the compactification scale, and the detailed superpartner spectroscopy will uncover the geometry of matter fields in extra dimensions and help discriminate between various possibilities for supersymmetry breaking.

Proton decay can occur in our theory via the bulk gauge interactions of the $X$ gauge boson through flavor mixing matrices. However, the resulting partial lifetime $\tau\left(p \rightarrow K^{+} \bar{\nu}\right) \approx$ $10^{37 \pm 2}$ years is probably too long to be reached by future experiments. Proton decay can also be mediated by brane kinetic operators, and these we estimate to give a lifetime of about $10^{34}$ years. The structure of the final states is very rich with comparable branching ratios to $e^{+} \pi^{0}, \mu^{+} \pi^{0}, e^{+} K^{0}, \mu^{+} K^{0}, \pi^{+} \bar{\nu}$ and $K^{+} \bar{\nu}$. Although the uncertainty in the lifetime is large as the coefficient for the relevant brane operator is not predicted, the branching ratios are all given in terms of a single unknown mixing parameter. Since gauge boson mediated proton decay does not involve an exchange of superparticles, these results are completely independent of supersymmetry breaking.

As stressed above, the requirements of a large top quark mass and proton longevity require a separation in location between the top quark and the up quark $-T_{3}$ must be on the brane and $T_{1}$ in the bulk. Introducing supersymmetry breaking by a twist in the boundary condition, this requires that $F_{1,2,3}$ share a common location, to avoid too large $\mu \rightarrow e$ and $\tau \rightarrow \mu$ transition rates. This immediately leads to a prediction of large neutrino mixing angles following from the see-saw generated neutrino mass matrix; both atmospheric and solar neutrino oscillations should result from large mixing angles. The Majorana mass for the right-handed neutrino arises from a brane-localized operator, and must have a size which is suppressed relative to the cutoff scale of our effective 5D theory. This suggests that the right-handed neutrino mass is protected 
by some symmetry, which we take to be the $U(1)_{X}$ remnant of $S O(10)$. The breaking of $U(1)_{X}$ gauge symmetry leads not only to right-handed neutrino masses, but also, when supersymmetry is broken, to the $\mu$ and $B$ parameters of the Higgs potential.

We have seen that a $U(1)_{R}$ symmetry is a critical feature of our theory, yet this symmetry is clearly broken by the supersymmetry breaking operators generated by the boundary conditions. Ultimately, the supersymmetry breaking will be spontaneous, arising from the vacuum expectation value of a field in the $5 \mathrm{D}$ supergravity multiplet. It therefore seems natural to assume that all breaking of $U(1)_{R}$ is also spontaneous, in which case there is an $R$ axion. This $R$ axion has a QCD anomaly and may therefore solve the strong $C P$ problem. We also find that its decay constant may be in an interesting range for the axion to be dark matter.

We have found that $S U(5)$ unification in 5D offers many advantages over unification in $4 \mathrm{D}$. While we have not addressed the origin of radius stabilization or matter localization, our effective field theory is remarkably simple; for example, the only non-trivial $S U(5)$ multiplets beyond the gauge multiplet are five-plets and ten-plets. Our theory is sufficiently constrained that it offers several avenues for experimental tests. We expect the first direct experimental signal for our theory to be the observation of events containing two "stable" charged particles

at the Tevatron or at LHC. These scalar taus have opposite charges if they arise from Drell-Yan production, but have equal probability of like charge and opposite charge combinations if they are produced from squark and gluino decays. It is likely that these charged scalar taus decay cosmologically to neutral axinos, which may contribute to dark matter of the universe.

\section{Acknowledgments}

Y.N. thanks the Miller Institute for Basic Research in Science for financial support. This work was supported in part by the Director, Office of Science, Office of High Energy and Nuclear Physics, of the U.S. Department of Energy under Contract DE-AC03-76SF00098, and in part by the National Science Foundation under grant PHY-00-98840. 


\section{References}

[1] L. J. Hall and Y. Nomura, Phys. Rev. D 64, 055003 (2001) arXiv:hep-ph/0103125.

[2] L. J. Hall and Y. Nomura, arXiv:hep-ph/0111068.

[3] Y. Kawamura, Prog. Theor. Phys. 105, 999 (2001) arXiv:hep-ph/0012125.

[4] S. Dimopoulos and H. Georgi, Nucl. Phys. B 193, 150 (1981);

N. Sakai, Z. Phys. C 11, 153 (1981).

[5] N. Sakai and T. Yanagida, Nucl. Phys. B 197, 533 (1982);

S. Weinberg, Phys. Rev. D 26, 287 (1982).

[6] A. Hebecker and J. March-Russell, Nucl. Phys. B 613, 3 (2001) arXiv:hep-ph/0106166.

[7] L. J. Hall, Y. Nomura and D. R. Smith, arXiv:hep-ph/0107331;

L. Hall, J. March-Russell, T. Okui and D. R. Smith, arXiv:hep-ph/0108161.

[8] Y. Nomura, Phys. Rev. D 65, 085036 (2002) arXiv:hep-ph/0108170.

[9] D. E. Groom et al. [Particle Data Group Collaboration], 2001 off-year partial update for the 2002 edition available on the PDG WWW pages (URL: http://pdg.lbl.gov/).

[10] R. Barbieri, L. J. Hall and Y. Nomura, arXiv:hep-ph/0106190.

[11] D. Marti and A. Pomarol, Phys. Rev. D 64, 105025 (2001) arXiv:hep-th/0106256;

D. E. Kaplan and N. Weiner, arXiv:hep-ph/0108001;

G. von Gersdorff and M. Quiros, Phys. Rev. D 65, 064016 (2002) arXiv:hep-th/0110132.

[12] T. Yanagida, in Proceedings of the Workshop on the Unified Theory and Baryon Number in the Universe, edited by O. Sawada and A. Sugamoto (KEK report 79-18, 1979), p. 95;

M. Gell-Mann, P. Ramond, and R. Slansky, in Supergravity, edited by P. van Nieuwenhuizen and D.Z. Freedman (North Holland, Amsterdam, 1979), p. 315.

[13] L. J. Hall, Y. Nomura and A. Pierce, arXiv:hep-ph/0204062.

[14] S. Weinberg, PhysicaA 96, 327 (1979);

A. Manohar and H. Georgi, Nucl. Phys. B 234, 189 (1984);

H. Georgi and L. Randall, Nucl. Phys. B 276, 241 (1986).

[15] Y. Nomura, D. R. Smith and N. Weiner, Nucl. Phys. B 613, 147 (2001) arXiv:hepph/0104041.

[16] M. S. Chanowitz, J. R. Ellis and M. K. Gaillard, Nucl. Phys. B 128, 506 (1977);

A. J. Buras, J. R. Ellis, M. K. Gaillard and D. V. Nanopoulos, Nucl. Phys. B 135, 66 (1978). 
[17] L. Randall and R. Sundrum, Nucl. Phys. B 557, 79 (1999) arXiv:hep-th/9810155.

[18] D. E. Kaplan, G. D. Kribs and M. Schmaltz, Phys. Rev. D 62, 035010 (2000) arXiv:hepph/9911293];

Z. Chacko, M. A. Luty, A. E. Nelson and E. Ponton, JHEP 0001, 003 (2000) arXiv:hepph/9911323.

[19] R. Barbieri, L. J. Hall and Y. Nomura, Nucl. Phys. B 624, 63 (2002) arXiv:hepth/0107004.

[20] L. J. Hall, H. Murayama and Y. Nomura, arXiv:hep-th/0107245.

[21] N. Arkani-Hamed, L. J. Hall, D. R. Smith and N. Weiner, Phys. Rev. D 63, 056003 (2001) arXiv:hep-ph/9911421;

N. Arkani-Hamed, T. Gregoire and J. Wacker, arXiv:hep-th/0101233.

[22] F. Gabbiani, E. Gabrielli, A. Masiero and L. Silvestrini, Nucl. Phys. B 477, 321 (1996) arXiv:hep-ph/9604387.

[23] Y. Okada, M. Yamaguchi and T. Yanagida, Prog. Theor. Phys. 85, 1 (1991);

J. R. Ellis, G. Ridolfi and F. Zwirner, Phys. Lett. B 257, 83 (1991);

H. E. Haber and R. Hempfling, Phys. Rev. Lett. 66, 1815 (1991).

[24] L. J. Hall, H. Murayama and N. Weiner, Phys. Rev. Lett. 84, 2572 (2000) arXiv:hepph/9911341].

[25] P. Langacker and N. Polonsky, Phys. Rev. D 52, 3081 (1995) arXiv:hep-ph/9503214.

[26] P. Langacker and N. Polonsky, Phys. Rev. D 47, 4028 (1993) arXiv:hep-ph/9210235;

M. Carena, S. Pokorski and C. E. Wagner, Nucl. Phys. B 406, 59 (1993) arXiv:hepph/9303202].

[27] T. Goto and T. Nihei, Phys. Rev. D 59, 115009 (1999) arXiv:hep-ph/9808255;

H. Murayama and A. Pierce, Phys. Rev. D 65, 055009 (2002) arXiv:hep-ph/0108104.

[28] R. Contino, L. Pilo, R. Rattazzi and E. Trincherini, Nucl. Phys. B 622, 227 (2002) arXiv:hep-ph/0108102.

[29] L. J. Hall, R. Rattazzi and U. Sarid, Phys. Rev. D 50, 7048 (1994) arXiv:hep-ph/9306309;

R. Hempfling, Phys. Rev. D 49, 6168 (1994);

M. Carena, M. Olechowski, S. Pokorski and C. E. Wagner, Nucl. Phys. B 426, 269 (1994) arXiv:hep-ph/9402253.

[30] A. Hebecker and J. March-Russell, arXiv:hep-ph/0204037.

[31] R. D. Peccei and H. R. Quinn, Phys. Rev. Lett. 38, 1440 (1977);

S. Weinberg, Phys. Rev. Lett. 40, 223 (1978);

F. Wilczek, Phys. Rev. Lett. 40, 279 (1978). 
[32] L. J. Hall, V. A. Kostelecky and S. Raby, Nucl. Phys. B 267, 415 (1986).

[33] R. Barbieri and L. J. Hall, Phys. Lett. B 338, 212 (1994) arXiv:hep-ph/9408406.

[34] R. Barbieri, L. J. Hall and A. Strumia, Nucl. Phys. B 445, 219 (1995) arXiv:hep$\mathrm{ph} / 9501334$.

[35] J. Hisano, T. Moroi, K. Tobe and M. Yamaguchi, Phys. Lett. B 391, 341 (1997) [Erratumibid. B 397, 357 (1997)] [arXiv:hep-ph/9605296].

[36] M. L. Brooks et al. [MEGA Collaboration], Phys. Rev. Lett. 83, 1521 (1999) arXiv:hepex/9905013.

[37] L.M. Barkov et al., research proposal to PSI (1999);

S. Ritt, in Proceedings of the 2nd Workshop on Neutrino Oscillations and their Origin, edited by Y. Suzuki et al. (World Scientific, Singapore, 2001), p. 245.

[38] F. Borzumati and A. Masiero, Phys. Rev. Lett. 57, 961 (1986);

J. Hisano, T. Moroi, K. Tobe, M. Yamaguchi and T. Yanagida, Phys. Lett. B 357, 579 (1995) arXiv:hep-ph/9501407;

J. Hisano, T. Moroi, K. Tobe and M. Yamaguchi, Phys. Rev. D 53, 2442 (1996) arXiv:hepph/9510309.

[39] U. Bellgardt et al. [SINDRUM Collaboration], Nucl. Phys. B 299, 1 (1988).

[40] J. Bernabeu, E. Nardi and D. Tommasini, Nucl. Phys. B 409, 69 (1993) arXiv:hepph/9306251, and references therein.

[41] T. Suzuki, D. F. Measday and J. P. Roalsvig, Phys. Rev. C 35, 2212 (1987).

[42] C. Dohmen et al. [SINDRUM II Collaboration.], Phys. Lett. B 317, 631 (1993).

[43] M. Bachman et al. [MECO Collaboration.], experimental proposal to Brookhaven National Laboratory AGS (1997).

[44] S. Ahmed et al. [CLEO Collaboration], Phys. Rev. D 61, 071101 (2000) arXiv:hepex/9910060].

[45] Y. Fukuda et al. [Super-Kamiokande Collaboration], Phys. Rev. Lett. 81, 1562 (1998) arXiv:hep-ex/9807003; Phys. Rev. Lett. 86, 5651 (2001) arXiv:hep-ex/0103032.

[46] Q. R. Ahmad et al. [SNO Collaboration], Phys. Rev. Lett. 87, 071301 (2001) [arXiv:nuclex/0106015; arXiv:nucl-ex/0204008.

[47] H. P. Nilles, M. Srednicki and D. Wyler, Phys. Lett. B 120, 346 (1983);

J. P. Derendinger and C. A. Savoy, Nucl. Phys. B 237, 307 (1984). 